Relocalización de la industria manufacturera en México en la apertura comercial, 1980-2014

Jorge Rafael Figueroa Elenes, Tomás Arroyo Parra y Aneliss Aragón Jiménez

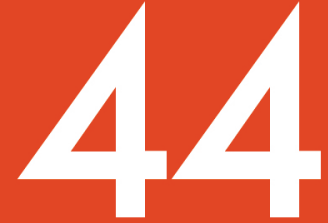

MARZO/ABRIL 2018

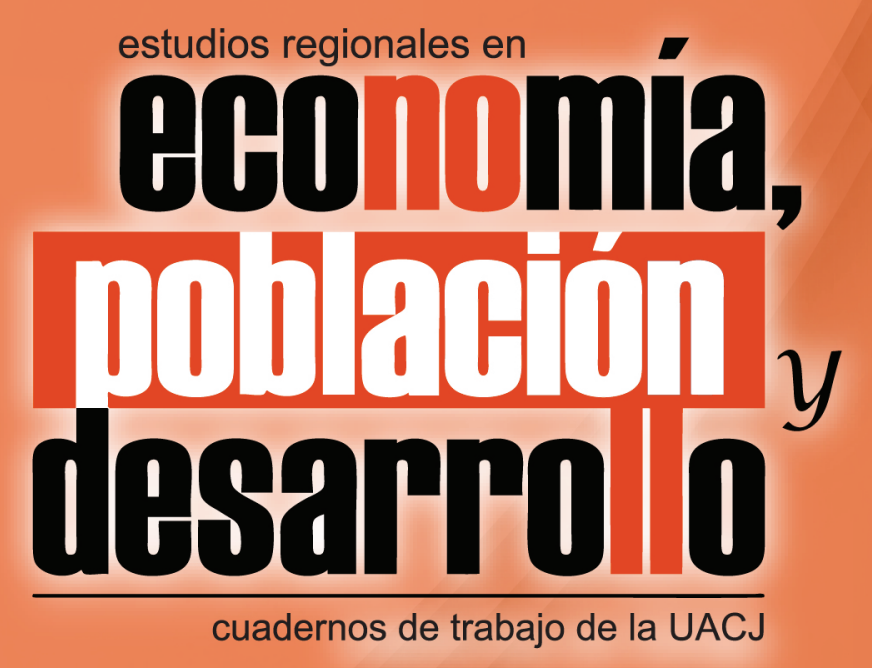




\title{
Relocalización de la industria manufacturera en México en la apertura comercial, 1980-2014
}

\author{
Jorge Rafael Figueroa Elenes, Tomás Arroyo Parra \\ y Aneliss Aragón Jiménez
}

UNIVERSIDAD AUTÓNOMA DE CIUDAD JUÁREZ 


\title{
UNIVERSIDAD AUTÓNOMA DE CIUDAD JUÁREZ \\ Instituto de Ciencias Sociales y Administración
}

\author{
Cuerpo Académico de Estudios Regionales en \\ Economía, Población y Desarrollo
}

Lic. Ricardo Duarte Jáquez

Rector

M.C. David Ramírez Perea

Secretario General

Mtro. Juan Ignacio Camargo Nassar

Director del Instituto de Ciencias

Sociales y Administración

Mtro. Ramón Chavira Chavira

Director General de Difusión

Cultura y Divulgación Científica

Dr. Luis Enrique Gutiérrez Casas

Coordinador General de

Investigación y Posgrado

\section{Dr. Jaime Alberto Arellano Quiroga \\ Coordinador del Cuerpo Académico de \\ Estudios Regionales en Economía, Población y Desarrollo}

Director y editor

Dr. Luis Enrique Gutiérrez Casas

Comité editorial

Sección internacional

Dra. Sofía Boza Martínez (Universidad de Chile, Chile)

Dra. Olga Biosca Artiñano (Glasgow Caledonian

University, Reino Unido)

Dra. Ángeles Sánchez Díez (Universidad Autónoma de

Madrid, España)

Dr. Thomas Fullerton Mankin (University of Texas at

E1 Paso, Estados Unidos)

Dr. Adrián Rodríguez Miranda (Universidad de la

República, Uruguay)

Sección local

(Universidad Autónoma de Ciudad Juárez)

Dra. Myrna Limas Hernández

Dra. Ikuho Kochi

Dr. Raúl Alberto Ponce Rodríguez

Dr. Isaac Leobardo Sánchez Juárez

Dr. Héctor Alonso Barajas Bustillos

Diseño de cubierta Abigail Bautista
Estudios Regionales en Economía, Población

y Desarrollo. Cuadernos de Trabajo de la UACJ

ISSN 2007-3739

Número 44. Marzo/Abril 2018

Relocalización de la industria manufacturera en

México en la apertura comercial, 1980-2014

Jorge Rafael Figueroa Elenes, Tomás Arroyo Parra

y Aneliss Aragón Jiménez

Universidad Autónoma de Ciudad Juárez

Estudios Regionales en Economía, Población y Desarrollo. Cuadernos de Trabajo de la UACJ

Año 8, No. 44 marzo - abril 2018, es una publicación bimestral editada por la Universidad Autónoma de Ciudad Juárez a través del Cuerpo Académico de Estudios Regionales en Economía, Población y Desarrollo del Instituto de Ciencias Sociales y Administración. Redacción: Avenida Universidad y H. Colegio Militar, Zona Chamizal s/n., C.P. 32300, Ciudad Juárez, Chihuahua, México. Teléfonos: (656) 688-38-00, ext. 3792. Correo electrónico: lgtz@uacj.mx.

Editor responsable: Luis Enrique Gutiérrez Casas. Reserva de derechos al uso exclusivo No. 04-2011-021713353900-102. ISSN 2007-3739, Impresa por Studio Los Dorados, calle Del Campanario, número 820-2, Santa Cecilia, C.P. 32350, Cd. Juárez, Chihuahua. Distribuidor: Subdirección de Gestión de Proyecto y Marketing Editorial. Ave. Plutarco Elías Calles 1210, Foviste Chamizal, C.P. 32310, Ciudad Juárez, Chihuahua. Este número se terminó de imprimir el 15 de febrero, 2018 con un tiraje de 120 ejemplares.

Los ensayos publicados son responsabilidad exclusiva de sus autores. Se autoriza la reproducción total o parcial bajo condición de citar la fuente.

\section{Registrada en:}

Publicación afiliada a la Red Iberoamericana de Estudios del Desarrollo

Universidad Autónoma de Ciudad Juárez

Ave Plutarco Elías Calles 1210

Foviste Chamizal, C.P. 32310

Ciudad Juárez, Chihuahua, México

www.uacj.mx

(C) Universidad Autónoma de Ciudad Juárez 


\title{
Relocalización de la industria manufacturera en México en la apertura comercial, 1980-2014
}

\author{
Jorge Rafael Figueroa Elenes*, Tomás Arroyo Parra** \\ y Aneliss Aragón Jiménez***
}

\begin{abstract}
Resumen
El propósito de este artículo es analizar los cambios en la localización y distribución del sector manufacturero en México. El estudio incluye una revisión del contexto de la industria manufacturera y la aplicación de técnicas de análisis regional tomando como año base a 1980 y año final al 2014. Se concluye que ha existido un fenómeno de relocalización manufacturera en México, los estados que conforman a la región del Bajío se constituyen como los más ganadores en materia manufacturera, así mismo se ha generado una reestructuración dentro del sector manufacturero, el subsector alimentario ha perdido gran peso en el total del sector, mientras que la maquinaria y el equipo se posiciona como la principal especialización manufacturera en las regiones del Bajío y del Norte de México.
\end{abstract}

Palabras clave: Industria manufacturera, localización industrial, distribución manufacturera.

\begin{abstract}
The purpose of this article is to analyze the changes in the location and distribution of the manufacturing sector in Mexico. The study includes a review of the context of the manufacturing industry and the application of regional analysis techniques based on the base year of 1980 and the end of 2014. It is concluded that there has been a phenomenon of manufacturing relocation in Mexico, the states that make up the Bajio region are the most winners in manufacturing, as well as a restructuring within the manufacturing sector, the food subsector has lost great weight in the total sector, while machinery and equipment is positioned as the main manufacturing specialization in the "Bajio" and Northern regions of Mexico.
\end{abstract}

Keywords: Manufacturing industry, industrial location, manufacturing distribution.

JEL Classification: 014, L6, R12.

- Recibido en: Noviembre de 2017.

- Aprobado en: Enero de 2018.

* Profesor de la Facultad de Ciencias Económicas y Sociales de la Universidad Autónoma de Sinaloa, Coordinador del Centro de Estudios sobre el Desarrollo Económico Local (CEDEL) y líder del Cuerpo Académico en Economía Aplicada. Correo electrónico: fijr@uas.edu.mx.

** Estudiante del Doctorado en Ciencias Sociales de la Facultad de Ciencias Económicas y Sociales de la Universidad Autónoma de Sinaloa. Colaborador del Centro de Estudios sobre el Desarrollo Económico Local (CEDEL) y del Cuerpo Académico en Economía Aplicada. Correo electrónico: tomasarroyo@facesuas.edu.mx. *** Estudiante del Doctorado en Ciencias Sociales de la Facultad de Ciencias Económicas y Sociales de la Universidad Autónoma de Sinaloa. Colaborador del Centro de Estudios sobre el Desarrollo Económico Local (CEDEL) y del Cuerpo Académico en Economía Aplicada. Correo electrónico: aneliss_aj23@hotmail.com. 


\section{$\rightarrow$ 1. Introducción.}

En los últimos setenta años, se han establecido tres modelos económicos en México: desarrollo estabilizador (1940-1970), desarrollo compartido (1970-1983) y el modelo de crecimiento hacia afuera (1983-actualidad). Estas estrategias modificaron la estructura productiva del país y, en particular, la industria manufacturera se constituyó como uno de los principales sectores que presentaron modificaciones debido a las nuevas políticas públicas (Huerta y Chávez, 2000).

El modelo de desarrollo estabilizador orientaba sus objetivos hacia alcanzar el desarrollo a través de la estabilidad macroeconómica, teniendo como principal característica el desarrollo de la industria nacional, a través del modelo de industrialización por sustitución de importaciones (ISI).

El modelo de desarrollo compartido enfocó sus esfuerzos a la inversión pública en infraestructuras productivas. Sin embargo, ante una caída abrupta en los precios internacionales del petróleo se hizo evidente que la política económica vigente no presentaba resultados favorables. Ante las tendencias crecientes de desigualdad del ingreso y de altos niveles de inflación derivados del proteccionismo comercial, se concluyó que el modelo económico no era apropiado frente a un mundo cada vez más globalizado (Huerta y Chávez, 2000).

Por lo tanto, el modelo de crecimiento hacia dentro, prevaleciente hasta entonces, se consideró agotado y el estado en turno se planteó la necesidad de sustituirlo por el que ha sido llamado modelo neoliberal. Así se dio por terminada la estrategia por sustitución de importaciones y la industrialización dirigida por el Estado y se avanzó hacia un plan de liberalización comercial, con el fin de atraer inversiones que se tradujeran en el establecimiento de industrias manufactureras en territorio nacional.

La nueva apertura comercial presentó un avance importante en 1986 con la adhesión de México al Acuerdo General sobre Aranceles y Comercio (GATT por sus siglas en inglés). De igual manera, el modelo neoliberal tuvo como principal evento la firma del Tratado del Libre Comercio con América del Norte (TLCAN) en 1992, entrando en vigor en 1994. A partir de la implementación del TLCAN se modificaron algunas de las principales variables macroeconómicas. Por ejemplo, la Inversión Extranjera Directa (IED) creció con un multiplicador de 6.6 veces, el empleo en el sector manufacturero se incrementó en 46 por ciento, mientras que el nivel de consumo de las familias aumentó solo a una tasa de 1.4 por ciento (Ruiz, 2015).

Lo cierto es que la situación económica del país parece ir en declive, en la década del 20002010 la fuerza de trabajo se incrementó en alrededor de 10 millones de personas, de las cuales sólo 3 millones se emplearon en trabajos formales. De igual manera, los ingresos han perdido poder adquisitivo, en 2001 el 10.3 por ciento de la población ocupada tenía ingresos de cinco o más salarios mínimos, mientras que para el 2013, sólo el 8.4 por ciento recibía ese nivel de ingresos (Cordera, 2015). 
La impresión general es que el incremento en el nivel de las exportaciones y la industria manufacturera ha sido ineficaz en generar un crecimiento económico sostenido en el país, debido a que sólo un número reducido de entidades federativas ha presentado modificaciones productivas en favor de las actividades que generan mayores niveles de valor agregado.

Ante este panorama, se considera de gran relevancia ahondar sobre este tema y conocer la dinámica del sector manufacturero a nivel estatal, tomando como referencia un periodo anterior a la apertura y uno posterior, pero no sólo esto, sino también es de suma importancia realizar un análisis de los subsectores manufactureros para complementar el estudio de nueva localización de las actividades industriales.

Dentro de este marco, el objetivo del presente estudio es señalar si existe una nueva localización manufacturera tomando como año base 1980 y como año final al 2014. Se analizan las nuevas especializaciones productivas en materia manufacturera que presentan los estados de México. De esta forma, se podrá establecer qué entidades federativas se han visto favorecidas en su nivel de desarrollo manufacturero, contrastando los cambios en el sector general y en los subsectores del mismo a nivel estatal.

\section{El contexto actual del sector manufacturero en México.}

Los efectos de la apertura comercial fueron diversos, y cada uno de ellos repercutió en el sector industrial. En el periodo de 1994-2008, las exportaciones aumentaron de 50 a 300 millones de dólares, un 75 por ciento corresponde a manufacturas. Por su parte, la IED se incrementó en casi 10 mil millones de dólares. A su vez, el tiempo requerido para la adopción de nuevas tecnologías provenientes de la región disminuyó en casi un año (Castañeda, 2010).

Se destaca que en 2006 se crea el programa para el Fomento de la Industria Manufacturera, Maquiladora y de Servicios de Exportación (IMMEX) con el objetivo de fortalecer el sector de las exportaciones mediante la capacidad de importar bienes e insumos necesarios para los procesos industriales sin realizar el pago de los impuestos de importación. Actualmente, acorde con datos de la Secretaría de Economía Nacional, las empresas adheridas al IMMEX conforman el 85 por ciento de las exportaciones de manufacturas en el país.

En el periodo reciente (2003-2014), acorde con datos del Instituto Nacional de Estadística y Geografía (INEGI), el total del sector manufacturero se ha incrementado a una tasa media de crecimiento de 2.16 por ciento, lo que refleja un aumento de 500 mil millones de pesos en 11 años. Se observa una caída importante en el producto manufacturero en el año $2009^{1}$, sin embargo, se experimenta una rápida recuperación en los años siguientes para concluir en el 2014 con un valor de más de 2 mil millones de pesos. 
Gráfica 1

Evolución del sector manufacturero

2003-2014

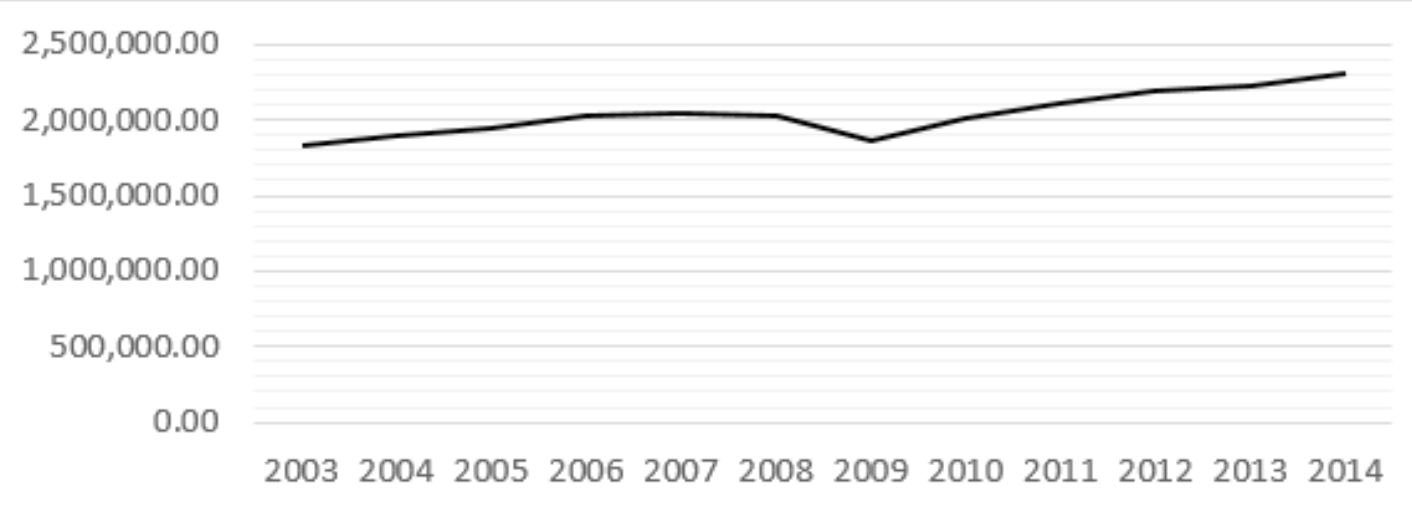

Fuente. Elaboración propia con datos del Banco de Información Económica del INEGI.

El subsector de la industria de maquinaria y equipo tiene una participación de 30 por ciento respecto al total del sector manufacturero, por lo que representa la industria de mayor relevancia dentro de las manufacturas. Así mismo, es la que ha experimentado mayor crecimiento en los últimos 11 años, con una tasa media de crecimiento de 4.84 por ciento.

\section{Gráfica 1}

\section{Evolución del subsector de maquinaria y equipo} 2003-2014

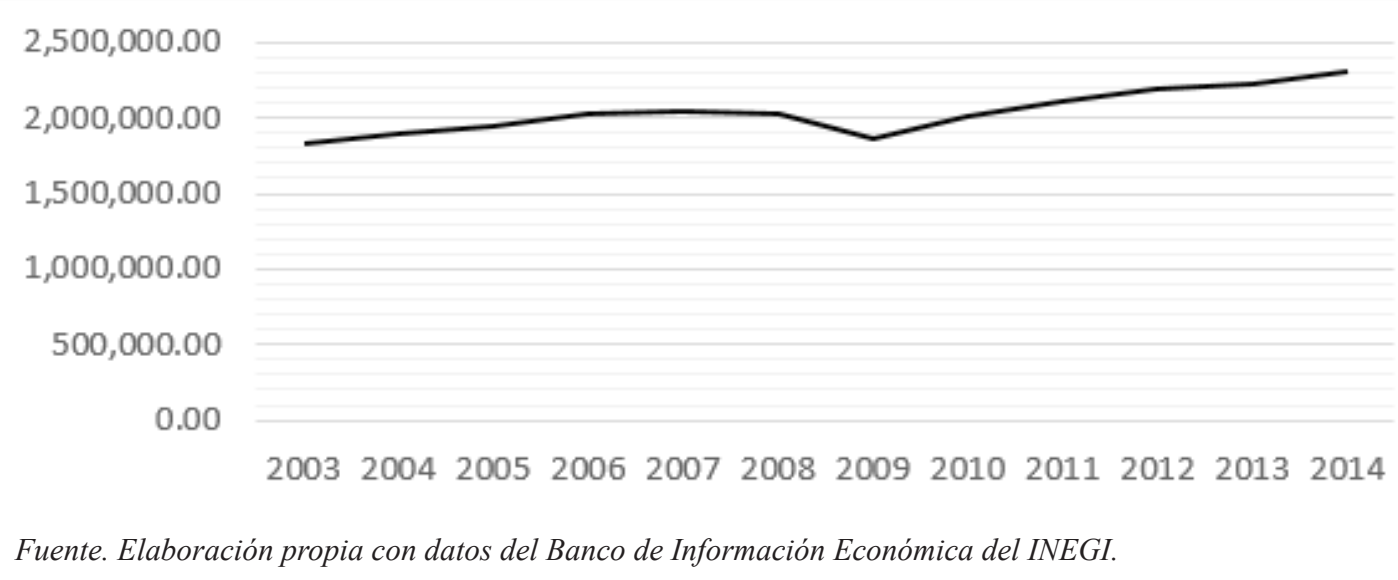

1 Como consecuencia de la crisis financiera en los Estados Unidos. 
La productividad del sector manufacturero en México apenas se ha incrementado en 6 puntos porcentuales, con respecto al año base (1993), en los últimos 11 años, lo que significa que, aunque el sector manufacturero ha crecido, la productividad del mismo se ha mantenido en cierta medida constante.

Actualmente, el empleo en el sector manufacturero representa el 16.38 por ciento del total del personal ocupado en el país, con alrededor de 8 millones de personas empleadas en enero de 2016.

Por otro lado, la producción de automóviles ${ }^{2}$ representa una parte fundamental del sector manufacturero. En los últimos meses del año 2016 se producen alrededor de 280 mil automóviles dentro de territorio mexicano. Se observa en la figura 3 un crecimiento pronunciado a partir del año de 1995, seguido por una crisis significativa en el año 2008, para posteriormente presentar una recuperación acelerada.

Gráfica 3

\section{Producción de automóviles en México}

1983-2016

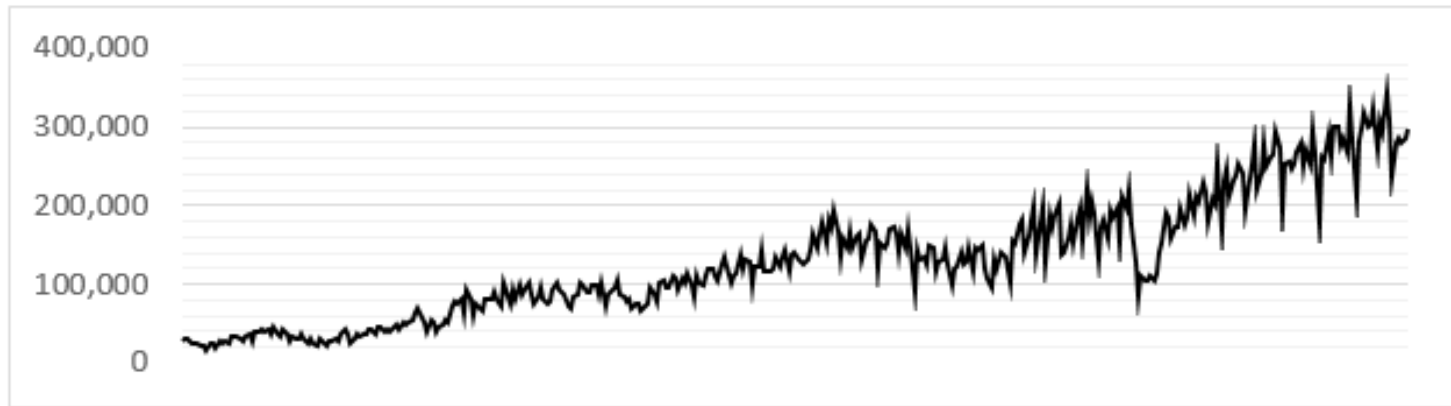

Fuente. Elaboración propia con datos del Banco de Información Económica del INEGI.

Para concluir esta sección, es importante mencionar que el número de establecimientos provenientes del programa IMMEX se ha mantenido relativamente constante, en alrededor de 5 mil establecimientos en el país.

\section{Estudios clásicos y recientes de la localización industrial.}

La teoría de la localización industrial ha evolucionado notablemente y su desarrollo es tal que sus planteamientos se encuentran incorporados en la corriente de la Nueva Geografía Económica (NGE). 
Se destacan como trabajos pioneros los elaborados por Von Thünen (1820), Weber (1909) y Marshall (1920), donde la localización con respecto al mercado y los costos de transporte son esenciales para reducir los costos.

Posteriormente, Christaller (1933) y Lösch (1940) introducen la idea de un espacio isotrópico $^{3}$ (igual en todas las direcciones) donde el área de mercado es creada a partir de la formación de los precios y de los costos de transporte. Más recientemente, Greenhut (1957) incorpora en sus postulados gran parte de la teoría microeconómica, al afirmar que para determinar la localización de las industrias es necesario analizar la competencia y la competitividad entre las empresas, concretamente se afirma que los factores económicos antes mencionados son determinantes para la elección de la localización.

A su vez, Aydalot (1985) afirma que la decisión de localizar una industria en un lugar determinado se encuentra en función de los recursos humanos y del nivel de infraestructura con el que cuente el territorio.

La teoría de la localización industrial tiene un estrecho vínculo con la teoría de la aglomeración, la cual hace referencia a la generación de externalidades, mismas que permiten la reducción de costos y el aprovechamiento de la proximidad de las industrias. En un atisbo visionario, Marshall (1920) interrelaciona dichas teorías afirmando que los sectores industriales pueden obtener ventajas en la producción al emplear la infraestructura de transporte, comunicación y servicios urbanos resultantes de los procesos de aglomeración. Asimismo, este autor señala la existencia de tres factores fundamentales que influyen en la concentración de la actividad industrial, estos son: el mercado de trabajo, los factores intermedios y la ósmosis tecnológica (Esqueda, 2013).

Continuando con la línea anterior, Félix (2003) señala las ideas de Ohlin (1933), quien propone cuatro categorías para definir la manera en que se presentan las economías de aglomeración:

$i$. Economías de escala internas a la empresa. Categoría que hace referencia a la producción de una gran cantidad de mercancía dentro de una misma industria y de la necesidad de una localización estratégica de la misma.

ii. Economías de Localización. Señala la necesidad de las empresas de agruparse para generar e ternalidades positivas.

iii. Economías de Urbanización. Señala los beneficios resultantes de una apropiada infraestructura urbana y de los conocimientos técnicos que existen en las grandes ciudades.

$i v$. Vinculación Inter-industrial. Hace referencia a la reducción en los costos de transporte resultado de las vinculaciones del insumo y el producto.

2 México mantiene un gran número de ensambladoras. Ford tiene plantas en los estados de Chihuahua, Sonora y el Estado de México. General Motors en Guanajuato. Honda en Jalisco. Volkswagen en Puebla, por señalar algunas.

$3 \mathrm{La}$ idea de los lugares centrales genera una diferencia entre aquellas ciudades denominadas como de primer orden y el resto con un orden más bajo (Hernández, 2009). 
Hoover (1948) define a las economías de aglomeración como la concentración de producción y de recursos, en donde las industrias cercanas se ven beneficiadas, y deben, además, elegir el lugar óptimo de localización en función de los mercados y los costos de transporte. Las aproximaciones mencionadas se acercan en cierta medida a las ideas de los clásicos de la localización industrial, al dar gran peso al costo del transporte, aunque se toma también como referencia a la generación de externalidades positivas, resultado de las economías de aglomeración.

La NGE desarrolla con mayor amplitud el concepto de economías de aglomeración, al explicar cómo en el interior de los territorios existen diversos factores que pueden concentrarse favoreciendo el establecimiento de las industrias. De acuerdo con Krugman y Livas (1992), se deben abarcar los dos conceptos de fuerza centrípeta y centrífuga dentro del marco de la NGE y de esta forma hacer un análisis completo de cómo los factores de aglomeración promueven y también pueden destruir el establecimiento de las industrias. La NGE toma como referencia el espacio geográfico del territorio y se encuentra respaldada por tres elementos: la teoría microeconómica, rendimientos crecientes a escala y un mercado de competencia imperfecto en el sector con las economías de escala (Félix, 2003).

En la búsqueda de estudios orientados al analizar de la localización espacial de la industria, se han encontrado trabajos como el realizado por Mori, et al. (2005), quienes construyen para la Unión Europea un índice de localización industrial, el cual permite señalar los cambios temporales que se hayan presentado en la distribución de la industria.

Holmes (2003) analiza la distribución espacial de las actividades económicas para Norteamérica y establece a las ventajas naturales, a la concentración del mercado y a la política gubernamental como características necesarias para entender la localización de las industrias. Por su parte, Shaver (2015) señala cómo las externalidades provocadas por las economías de aglomeración tienden a influir en la localización de las industrias.

Mendoza y Pérez (2007) vinculan los factores de la aglomeración con los encadenamientos de las industrias. Se encuentra para México que se ha presentado un cambio en la localización de la industria manufacturera en el periodo de 1980-2003. Hernández (2007) reafirma estos planteamientos encontrando que, a partir de la apertura comercial, se presenta una disminución en la concentración industrial en los estados del centro, golfo, sur y pacífico del país, mientras que los estados del norte han incrementado sus niveles de especialización.

Germán-Soto y Escobedo (2010) realizan un análisis de la evolución de la industria entre las entidades de México durante el periodo de 1960-1998, para ello utilizan la técnica de la econometría espacial y llegan a la conclusión de que existen agrupaciones de estados pobres en el sur del país y entidades más desarrolladas en el norte como consecuencia de la localización industrial. En el mismo orden de ideas, De León-Arias y Parra-De la Torre (2011) exploran las características 
de la dinámica de la productividad manufacturera por entidad federativa en México, logrando identificar tres grupos de entidades en función de su desempeño productivo: uno de las ganadoras, otro de las perdedoras y un tercero conformado por el resto del país.

\section{Las técnicas de análisis regional.}

Los objetivos del presente estudio buscan identificar las modificaciones en el patrón de localización de la industria manufacturera en México y estudiar las nuevas especializaciones productivas en la misma.

Existen diversas técnicas de análisis estructural entre las que se destacan la Matriz de Insumo producto de Leontief (1951), la novedosa teoría de grafos, los modelos de Pasinetti (1973) y Sraffa (1960), la econometría clásica y la econometría espacial, entre otros.

Sin embargo, las denominadas Técnicas de Análisis Regional (TAR), ofrecen ventajas relativas con respecto al resto de técnicas para el estudio de cambios estructurales, ya que combinan los elementos de tiempo y espacio para realizar un análisis más completo. Además, teniendo un año base y un año final, con las TAR es posible identificar los cambios estructurales que han existido en la industria manufacturera por entidad federativa y conocer si la distribución de la misma se ha mantenido con el paso del tiempo, o si por el contrario, se ha configurado un nuevo patrón de localización en el año final de estudio.

Las bondades de las TAR permiten analizar los sectores productivos, pero de igual manera, facilitan adentrarnos en los subsectores de determinada actividad para, de esta manera, investigar los elementos claves que han experimentado cambios estructurales en los territorios. Por lo tanto, las TAR se constituyen como una técnica fundamental y apropiada para responder a las preguntas de esta investigación.

Las TAR surgen en investigaciones realizadas en los Estados Unidos, donde se les ha otorgado gran importancia a los estudios territoriales, como lo muestran los trabajos de Isard (1960 y 1998), Hoover (1985) y Hayter (1997). De igual manera, la CEPAL desarrolló gran interés en el uso de las TAR, expresado principalmente por los trabajos de Boisier (1980) y Lira y Quiroga (2003).

Actualmente, los estudios que involucran los elementos espaciales han desarrollado una mayor importancia, por lo que las TAR se han convertido en una herramienta fundamental de análisis económico. Inclusive en los últimos años se han desarrollado nuevos coeficientes, los cuales se han adoptado como elementos clave de las TAR. Así pues, esta herramienta se encuentra en constante evolución.

Las TAR constituyen un paquete de técnicas para el diagnóstico de las regiones, las cuales, permiten dar respuesta a las interrogantes sobre las actividades en las que se especializa un territo- 
rio, el tamaño de dichas actividades, el grado de diversificación de la economía y en qué región se concentra el desarrollo de determinado sector (Méndez y Lloret, 2004).

Antes de iniciar la aplicación de los coeficientes de las TAR, es necesario seleccionar cuáles serán las unidades espaciales a estudiar y determinar los sectores que se analizarán.

Comúnmente, los datos recopilados se organizan a través de una matriz, las filas contienen la información de los sectores, mientras que las columnas hacen referencia a las regiones o territorios utilizados (Méndez y Lloret, 2004).

Tabla 1

Matriz principal para el uso de las TAR

\begin{tabular}{|c|c|c|c|c|c|c|}
\hline $\begin{array}{r}\text { REG } \\
\text { SEC }\end{array}$ & $\mathbf{R}_{1}$ & $\mathbf{R}_{2}$ & .............. & $\mathbf{R}_{\mathbf{j}}$ & $\mathbf{R}_{\mathrm{m}}$ & $\sum_{j=1}^{n} S E C$ \\
\hline $\mathbf{S}_{1}$ & $V_{11}$ & $V_{12}$ & ................. & $\mathbf{V}_{1 j}$ & $V_{l m}$ & $V_{1 j}$ \\
\hline $\mathbf{S}_{2}$ & $V_{21}$ & $V_{22}$ & ................. & $V_{2 j}$ & $\mathrm{~V}_{2 \mathrm{~m}}$ & $V_{2 j}$ \\
\hline $\mathbf{S}_{3}$ & $V_{31}$ & $V_{32}$ & ............. & $V_{3 j}$ & $\mathrm{~V}_{3 \mathrm{~m}}$ & $V_{3 j}$ \\
\hline$\cdots$. & $\cdots \cdot$ & $\cdots$. & ................ & $\cdots \cdot$ & $\cdots$. & $\cdots$. \\
\hline $\mathbf{S}_{\text {I }}$ & $V_{\text {il }}$ & $V_{i 2}$ & ................ & $\mathbf{V}_{\mathrm{ij}}$ & $V_{\text {im }}$ & $\mathbf{V}_{\mathrm{ij}}$ \\
\hline $\mathbf{S}_{\mathbf{n}}$ & $V_{n l}$ & $V_{n 2}$ & ................. & $V_{n j}$ & $V_{n m}$ & $V_{n j}$ \\
\hline$\sum_{i=1}^{n} R E G$ & $\mathrm{~V}_{\text {il }}$ & $\mathrm{V}_{\mathrm{i} 2}$ & ............ & $\mathbf{V}_{\mathrm{ij}}$ & $\mathbf{V}_{\text {im }}$ & $\mathbf{V}_{\mathrm{sr}}$ \\
\hline
\end{tabular}

Fuente: Recuperado de: Boisier (1980).

\section{Donde:}

$S_{i} \quad$ : Sector o rama de actividad.

$R_{j} \quad$ : Región o entidad geográfica en general.

$V \quad$ : Variable de análisis.

$V_{i j} \quad:$ Valor de la variable V correspondiente al sector " $i$ " $y$ región " $j$ ". 


\section{$V_{s j} \quad: \sum_{j=1}^{n} V_{i j}$, Valor de V correspondiente al total sectorial (sector $i$ ). \\ $V_{i r} \quad: \sum_{i=1}^{n} V_{i j}$, Valor de V correspondiente al total regional (región $j$ ). \\ $V_{s r} \quad$ : Valor de V correspondiente al total global (suma sectorial y regional).}

Para proceder con el uso de las TAR son necesarios dos periodos, denominados año base (inicial) y año dado (final). Para el ejercicio de análisis se toma el periodo de 1980-2014 y se plantea utilizar el análisis de las TAR en dos niveles. En primer lugar, las matrices construidas contendrán información referente al PIB de México, así el área de las columnas corresponde al total de las entidades federativas del país, mientras que las filas contienen los sectores en los que se divide el PIB nacional. En segundo lugar, se analizan únicamente los subsectores que conforman el sector de la industria manufacturera para el conjunto de las entidades federativas de México en el mismo periodo.

Para realizar la comparación y cálculo entre ambas matrices es necesario homogeneizar los sectores y las medidas que se utilizan. Por lo que resulta indispensable modificar las bases de datos correspondientes al año 1980 y 2014, para de ese modo obtener los mismos sectores y subsectores productivos. De igual manera, los datos deben estar calculados a precios constantes con el mismo año base, por lo que se utiliza un deflactor del $\mathrm{PIB}^{4}$.

Dentro del primer análisis, las matrices propuestas incluyen los siguientes sectores: agropecuario, minería, industria manufacturera, construcción, electricidad, comercio, transportes, servicios financieros y servicios comunales.

Cabe señalar que aunque la información hace referencia al conjunto de los sectores productivos que conforman el PIB, para los propósitos de la investigación, el análisis de los coeficientes se concentra únicamente en la evolución de la industria manufacturera. En el segundo análisis, los subsectores de la industria manufacturera a estudiar son: alimentario, bebidas y tabaco, insumos textiles, prendas de vestir, madera, papel, derivados del petróleo, minerales no metálicos, metálicos básicos, maquinaria y equipo, muebles y otros ${ }^{5}$.

Las TAR están conformadas por diversos coeficientes que tienen distintas finalidades y objetivos, sin embargo, en la presente investigación se utilizan únicamente aquellos que permitan observar cambios en la estructura productiva y en la distribución de la industria manufacturera. Todas las definiciones de los coeficientes de las TAR presentadas en este texto se han obtenido en base a los trabajos de investigación de Méndez y Lloret (2004), Boisier (1980) y Lira y Quiroga (2003).

4 La homogenización de los sectores se realizó de acuerdo a los existentes en 1980, mientras que para convertir los valores a precios constantes se utilizó un deflactor del PIB obtenido en Index Mundi.

5 Así mismo, fue necesario homogenizar los subsectores de acuerdo a los que existían en 1980. 
Participación del Sector en la Región.

El primer coeficiente mide el porcentaje que representa determinado sector (i) en la actividad total de la misma región $(j)$.

\section{a. Participación de la región en el Sector.}

Aunque el presente indicador es similar al anterior, este busca captar la participación de determinado sector de una región (i), en el total sectorial del contexto nacional.

\section{b. Cociente de Localización (Qij).}

Mediante el cociente de localización es posible vincular los cálculos de la participación del sector en la región y la participación total sectorial en el total global. Para realizar la interpretación de este indicador, se tiene que valores mayores a 1 indican especialización en determinado sector, debido a que se demuestra que existe un peso mayor de determinado sector en la región, que el mismo sector en el total de la suma de las economías.

$$
Q_{i j}=\left[\left(V_{i j} / \Sigma_{i} V_{i j}\right) /\left(\Sigma_{j} V_{i j} / \Sigma_{i} \Sigma_{j} V_{i j}\right)\right]
$$

c. Base Económica y Multiplicadores (Xij) y (Mi).

El cálculo de este indicador está representado por la siguiente expresión:

$$
X_{i j}=V_{i j}-\left(V_{i j} / Q_{i j}\right)
$$

Donde Xij se interpreta como la producción exportable del sector (i) de la entidad (j).

\section{d. Cociente de Variación (rVij).}

Este indicador también puede ser reconocido como una tasa de crecimiento, donde para realizar el cálculo se obtiene el cociente del valor de determinado sector en el año dado y el mismo sector en el año base, por lo que se logra captar si ha existido crecimiento o decrecimiento de un sector.

e. Coeficiente de Reestructuración (CRr). 
El presente indicador permite captar cambios en la estructura de las regiones teniendo como periodo al año base y el año dado. Los resultados de este coeficiente varían solamente entre 0 y 1 . Cuando se tiene que el coeficiente toma valor de 0 significa que los cambios en la estructura son nulos, mientras que, si el coeficiente se acerca en mayor medida a 1 hace referencia a una reestructuración de la región.

$$
C R r=\left(\frac{1}{2}\right) * \Sigma_{j}\left\{A B S\left[V_{i j}(t) / \Sigma_{i} V_{i j}(t)-V_{i j}(0) / \Sigma_{i} V_{i j}(0)\right]\right\}
$$

\section{f. Coeficiente de redistribución (CRs).}

El último indicador de las TAR que convendrá utilizar hace referencia a la dinámica de distribución de un sector en el tiempo. En este caso, se interpreta de modo que mientras más grande sea el coeficiente, se trata del sector donde más se ha modificado el peso que éste tiene en las entidades.

$$
C R S=\left(\frac{1}{2}\right) * \Sigma_{j}\left\{A B S\left[V_{i j}(t) / \Sigma_{j} V_{i j}(t)-V_{i j}(0) / \Sigma_{j} V_{i j}(0)\right]\right\}
$$

Mediante estos coeficientes se busca realizar un análisis completo de la localización del sector manufacturero y de los cambios en su composición.

\section{Relocalización y redistribución del sector manufacturero en México.}

El primer coeficiente en mención muestra el porcentaje de participación de la industria manufacturera en el total del producto de la misma entidad. Para el año 1980, las entidades que presentaban mayor participación industrial en sus economías eran el estado de México (37.89), Nuevo León (34.78), Querétaro (33.97), Hidalgo (33.2) y Coahuila (26.8), respectivamente.

Mientras que, como se observa en la tabla 2, para el año 2014, se encuentran cambios significativos en este indicador, ya que se tiene que entidades federativas como Coahuila (46.13), Aguascalientes (38.07), Guanajuato (34.24) y San Luis Potosí (30.45) han sido las que han presentado una mayor transformación en su aparato productivo en favor de la industria manufacturera.

Se destaca que el Estado de México ha perdido 8 posiciones, Nuevo León 6 posiciones y Jalisco 5 posiciones en el ranking de participación manufacturera. De igual manera, se adelanta una transformación productiva, principalmente en la zona del Bajío ${ }^{6} \mathrm{y}$, en un segundo plano, en los estados del norte ${ }^{7}$ de México. 
Tabla 2

Ranking de participación industrial en el total nacional 2014

\begin{tabular}{|c|c|c|c|}
\hline Ranking & Estado & Valor & Posiciones \\
\hline 1 & Coahuila & 46.13 & +4 \\
\hline 2 & Aguascalientes & 38.07 & +17 \\
\hline 3 & Guanajuato & 34.24 & +11 \\
\hline 4 & Tlaxcala & 33.34 & +4 \\
\hline 5 & Querétaro & 32.09 & -2 \\
\hline 6 & Hidalgo & 31.81 & -2 \\
\hline 7 & San Luis Potosí & 30.45 & +4 \\
\hline 8 & Nuevo León & 27.85 & -6 \\
\hline 9 & México & 25.88 & -8 \\
\hline 10 & Morelos & 25.59 & 0 \\
\hline 11 & Puebla & 25.38 & -3 \\
\hline 12 & Chihuahua & 24.38 & +8 \\
\hline 13 & Baja California & 23.53 & +2 \\
\hline 14 & Jalisco & 22.79 & -5 \\
\hline 15 & Veracruz & 22.27 & -3 \\
\hline & & & \\
\hline
\end{tabular}

Fuente. Elaboración propia con datos del Banco de Información Económica del INEGI.

El segundo indicador hace mención a la participación del sector industrial en el total nacional del mismo sector. Se observan grandes modificaciones en el periodo de 1980-2014. La Ciudad de México ha perdido más de 20 puntos de su participación en el contexto nacional ya que se ha enfocado en los sectores de comercio, transporte y financiero. De igual manera, el Estado de México ha reducido su participación en cerca de 7 puntos porcentuales.

Se destacan por su crecimiento en 2014 entidades como Coahuila, Guanajuato, Chihuahua, Aguascalientes, Tamaulipas y Sonora. Cabe señalar, que este indicador puede no reflejar un cambio radical en las economías de las entidades federativas debido a que muchas de ellas son economías

6 La región del Bajío se encuentra integrada por los estados de Aguascalientes, Querétaro, Guanajuato, Zacatecas y parte de Jalisco.

7 Se entiende por norte de México a las entidades federativas que conforman la frontera con Estados Unidos, Baja California, Sonora, Chihuahua, Nuevo León, Coahuila y Tamaulipas. 
pequeñas en comparación con estados como Nuevo León o la Ciudad de México, por lo que su participación en el contexto nacional es moderada.

Tabla 3

\section{Ranking de participación industrial en el total nacional}

$1980-2014$

\begin{tabular}{|c|c|c|c|c|c|}
\hline \multicolumn{5}{|c|}{1980} & \multicolumn{3}{|c|}{2014} \\
\hline Ranking & Estado & Valor & Ranking & Estado & Valor \\
\hline 1 & Ciudad de México & 29.45 & 1 & México & 11.91 \\
\hline 2 & México & 18.07 & 2 & Nuevo León & 10.47 \\
\hline 3 & Nuevo León & 9.10 & 3 & Coahuila & 8.23 \\
\hline 4 & Jalisco & 6.66 & 4 & Guanajuato & 7.26 \\
\hline 5 & Quintana Roo & 5.57 & 5 & Jalisco & 7.26 \\
\hline 6 & Veracruz & 5.27 & 6 & Ciudad de México & 6.88 \\
\hline 7 & Puebla & 3.78 & 7 & Veracruz & 5.87 \\
\hline 8 & Coahuila & 3.10 & 8 & Puebla & 4.09 \\
\hline 10 & Guanajuato & 2.37 & 9 & Querétaro & 3.57 \\
\hline
\end{tabular}

Fuente. Elaboración propia con datos del Banco de Información Económica del INEGI.

Para observar de una mejor manera los cambios en las estructuras productivas de los estados, se observa el cociente de localización, que señala la especialización productiva que tienen las economías en ciertos sectores. La región del Bajío se constituye como el territorio que ha presentado mayor transformación en su especialización manufacturera, de igual manera pero en segundo plano, se observa un alto nivel de especialización manufacturera en el norte del país.

En concreto, las entidades que presentaban mayor especialización en el sector manufacturero en 1980 eran: Estado de México, Nuevo León, Querétaro, Hidalgo, Coahuila, Puebla, Ciudad de México, Tlaxcala, Jalisco y Morelos, respectivamente.

Debido a los cambios en política económica, apertura comercial, localización geográfica, o bien, a planes internos de las entidades federativas, se observa que para 2014 los estados que se encuentran mayormente especializados en el sector manufacturero son: Coahuila, Aguascalientes, Guanajuato, Tlaxcala, Querétaro, Hidalgo, San Luis Potosí, Nuevo León, Estado de México y Morelos, respectivamente.

El siguiente indicador, denominado base económica, señala los sectores en los que las entidades federativas presentan material exportable. Los mayores cambios se presentaron en las entidades que han modificado su cociente de localización. Entidades como Aguascalientes, Baja 
California, Guanajuato y Sonora, no presentaban material manufacturero exportable en los ochenta, mientras que para 2014 la mayor concentración del indicador se encuentra en el sector manufacturero, para dichas entidades.

\section{Mapa 1}

\section{Cociente de Localización}

1980-2014

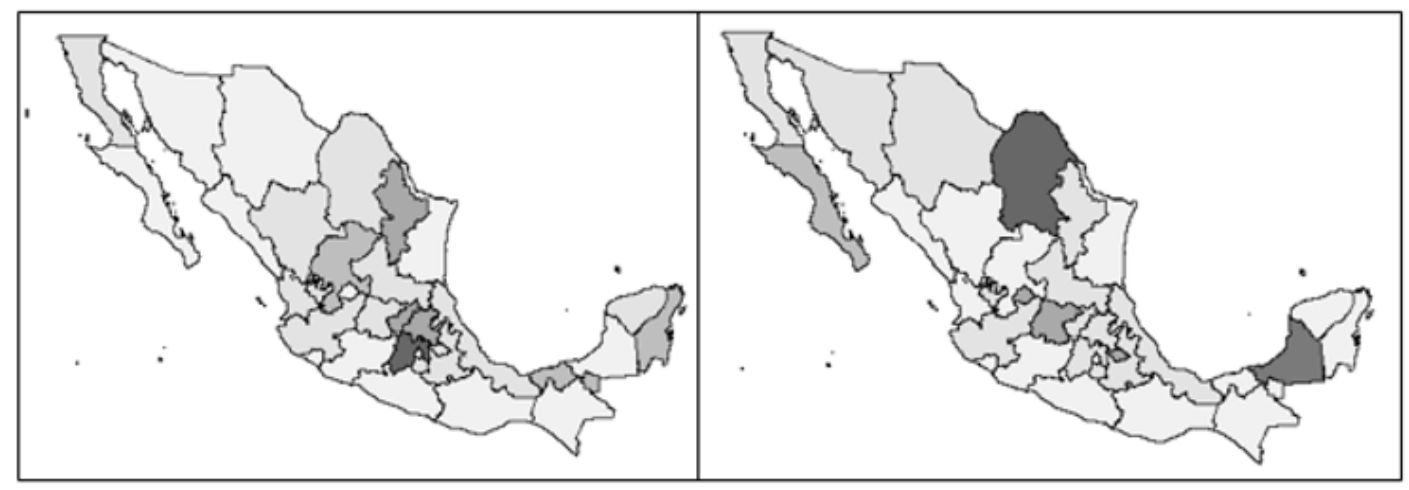

Nota: Los estados en tono más oscuro son los más industrializados. En la izquierda se encuentra la situación de 1980 y ala derecha de 2014.

Fuente: Elaboración propia mediante Geoda.

Lo anterior, hace referencia no sólo a un cambio moderado en la localización industrial, sino también a que en los últimos 34 años las modificaciones en la especialización de las entidades del Bajío y del norte de México ha sido completa, y se ha conformado un corredor industrial en el Bajío-Norte del país, el cual ha aprovechado las ventajas que se generan a partir de la aglomeración de actividades industriales en una región.

El siguiente grupo de indicadores no permiten una comparación entre año inicial (1980) y año final (2014). Sin embargo, utilizan ambos periodos para construir un análisis de mayor complejidad.

En primer lugar, el cociente de variación nos señala cuáles entidades han tenido una mayor tasa de crecimiento en el sector manufacturero. 
Tabla 4

\section{Ranking respecto al cociente de variación industrial}

1980-2014

\begin{tabular}{|c|c|c|c|c|c|c|c|}
\hline Ranking & Estado & CLAVE & Valor & Ranking & Estado & CLAVE & Valor \\
\hline 1 & Aguascalientes & AGS & 14.01 & 11 & Chihuahua & CHIH & 4.48 \\
\hline 2 & Quintana Roo & QR & 11.43 & 12 & Tamaulipas & TAMPS & 3.99 \\
\hline 3 & Zacatecas & ZAC & 8.41 & 13 & Michoacán & MICH & 3.90 \\
\hline 4 & Guanajuato & GTO & 7.15 & 14 & Tabasco & TAB & 3.77 \\
\hline 5 & Coahuila & COAH & 6.20 & 15 & Yucatán & YUC & 3.61 \\
\hline 6 & Sonora & SON & 6.07 & 16 & Morelos & MOR & 3.30 \\
\hline 7 & Querétaro & QRO & 5.94 & 17 & Durango & DGO & 3.24 \\
\hline 8 & San Luis Potosín & SLP & 5.15 & 18 & Oaxaca & OAX & 3.21 \\
\hline 9 & Tlaxcala & TLAX & 4.60 & 19 & Hidalgo & HGO & 2.87 \\
\hline 10 & Baja California & BC & 4.48 & 20 & Colima & COL & 2.86 \\
\hline
\end{tabular}

Fuente. Elaboración propia con datos del Banco de Información Económica del INEGI.

Se observa que, en el periodo de estudio, el mayor crecimiento manufacturero se ha dado principalmente en las entidades que conforman la región del Bajío. Así mismo, destacan las entidades federativas de Coahuila y Sonora en la frontera norte, así como también Quintana Roo.

Para concluir con el primer análisis, se describen los coeficientes de reestructuración y de redistribución, que muestran aquellas entidades en las que ha existido una mayor reestructuración productiva.

El cociente de redistribución permite realizar el análisis de cambio estructural a nivel nacional. Se observa que la minería es el sector que ha presentado mayores modificaciones positivas en su estructura debido a los descubrimientos de los pozos petroleros. Por su parte, el sector manufacturero se encuentra en segundo lugar a nivel nacional en lo referente a su modificación en sentido positivo, seguido del sector eléctrico, construcción, transporte, agropecuario, comunal y financiero.

En la tabla 5 es posible observar las entidades que han presentado una mayor reestructuración en favor del sector manufacturero, concluyéndose que las entidades federativas que conforman el Bajío han presentado un notable cambio estructural en sus economías. La industria manufacturera se ha convertido en un sector clave y en uno de los principales factores de su crecimiento. De igual manera, en las entidades del norte del país ha ganado peso el sector manufacturero, ubicándose el estado de Coahuila como el principal ganador en materia industrial. A su vez, se observa una pérdida de peso de la industria manufacturera en el centro y sur del país. 
Tabla 5

Ranking respecto al cociente de reestructuración y de redistribución 1980-2014

\begin{tabular}{|c|c|c|c|c|c|}
\hline \multicolumn{3}{|c|}{ Reestructuración } & \multicolumn{3}{c|}{ Redistribución } \\
\hline Ranking & Estado & Valor & Ranking & Estado & Valor \\
\hline 1 & Aguascalientes & 0.230 & 1 & Coahuila & 0.051 \\
\hline 2 & Coahuila & 0.193 & 2 & Guanajuato & 0.049 \\
\hline 3 & Guanajuato & 0.156 & 3 & Querétaro & 0.022 \\
\hline 4 & Sonora & 0.100 & 4 & Sonora & 0.020 \\
\hline 5 & Tlaxcala & 0.094 & 5 & Aguascalientes & 0.020 \\
\hline 6 & Chihuahua & 0.094 & 6 & Chihuahua & 0.017 \\
\hline 7 & San Luis Potosín & 0.089 & 7 & San Luis Potosí & 0.016 \\
\hline 8 & Zacatecas & 0.064 & 8 & Baja California & 0.016 \\
\hline 10 & Baja California & 0.059 & 9 & Nuevo León & 0.014 \\
\hline & Tamaulipas & 0.052 & 10 & Tamaulipas & 0.013 \\
\hline
\end{tabular}

Fuente. Elaboración propia con datos del Banco de Información Económica del INEGI.

Si bien se reconoce un cambio en la distribución del sector manufacturero hacia el Bajío y el norte del país, es necesario determinar en qué actividades se han especializado las entidades con altos índices de producto manufacturero.

Para el segundo análisis, debido a que las bases de datos ofrecen una gran cantidad de información, el análisis se concentra en las entidades federativas que han presentado transformaciones en sus aparatos productivos en favor de la industria manufacturera. La participación de los subsectores en el total manufacturero de los estados de México, nos indica que para el año de 1980, gran parte de las entidades federativas estudiadas destinaban un mayor peso a la industria de los alimentos, inclusive en el indicador nacional, el 37 por ciento del sector manufacturero estaba representado por la industria alimentaria. 
Relocalización de la industria manufacturera en México... • Jorge Rafael Figueroa Elenes, Tomás Arroyo Parra y Aneliss Aragón Jiménez

Tabla 6

\section{Participación de los subsectores en el total del producto manufacturero}

1980

\begin{tabular}{|c|c|c|c|c|c|c|c|c|c|c|c|}
\hline & AGS & BC & COAH & GTO & JAL & MEX & NL & QRO & SLP & SON & TAMPS \\
\hline Alimentos & 56.30 & 46.70 & 14.87 & 23.96 & 37.99 & 10.10 & 21.68 & 38.72 & 53.14 & 57.45 & 25.49 \\
\hline Textiles & 21.21 & 10.55 & 10.70 & 28.91 & 18.87 & 13.17 & 5.58 & 7.79 & 8.13 & 14.04 & 7.73 \\
\hline Madera & 1.25 & 4.62 & 1.19 & 0.99 & 4.33 & 2.20 & 1.38 & 0.48 & 4.96 & 3.08 & 2.07 \\
\hline Papel & 0.84 & 2.51 & 0.91 & 1.08 & 4.55 & 6.52 & 5.04 & 2.70 & 4.48 & 1.77 & 2.27 \\
\hline Químicos & 1.47 & 5.15 & 4.39 & 33.00 & 11.32 & 20.46 & 14.43 & 0.76 & 4.95 & 6.35 & 50.57 \\
\hline $\begin{array}{c}\text { Minería no } \\
\text { metálica }\end{array}$ & 3.56 & 3.93 & 9.26 & 4.47 & 7.38 & 6.81 & 12.28 & 3.76 & 11.22 & 3.73 & 3.25 \\
\hline Metálicos & 5.79 & 1.51 & 33.62 & 0.19 & 1.50 & 5.88 & 15.92 & 2.88 & 6.64 & 0.65 & 6.42 \\
\hline Maquinaria & 15.14 & 24.74 & 24.06 & 6.66 & 10.55 & 31.87 & 22.71 & 44.92 & 5.28 & 11.64 \\
\hline Otros & 0.17 & 1.77 & 1.00 & 0.73 & 3.52 & 3.00 & 0.97 & 0.88 & 1.21 & 1.27 & 1.43 \\
\hline
\end{tabular}

Fuente. Elaboración propia con datos del Banco de Información Económica del INEGI.

En la tabla 6 se observa que, en 1980, las entidades que actualmente se encuentran industrializadas seguían el patrón nacional, es decir, destinaban el mayor porcentaje de su producto manufacturero al subsector de la industria alimentaria. Entre los estados que no eran parte de esta tendencia se encontraban Coahuila, con una mayor aportación al subsector de la industria metálica, el Estado de México, con especialización en la industria química y de maquinaria, Querétaro quien tenía mayor participación en maquinaria y Tamaulipas que destinaba mayor porcentaje a la industria Química.

Para el año 2014 se observan cambios significativos en las entidades seleccionadas, ya que en gran parte de ellas la mayor participación de la industria alimentaria se ha trasladado a la industria de maquinaria y metálica. Sobresalen por su modificación productiva los estados de Aguascalientes, Baja California, San Luis Potosí, Guanajuato y Sonora. Para ampliar el análisis e iniciar la consideración de las nuevas especializaciones en los subsectores manufactureros, se presenta la tabla con los resultados del cociente de localización (Tabla 7). 
Tabla 7

\section{Participación de los subsectores en el total del producto manufacturero}

$$
2014
$$

\begin{tabular}{|c|c|c|c|c|c|c|c|c|c|c|c|}
\hline & AGS & BC & COAH & GTO & JAL & MEX & NL & QRO & SLP & SON & TAMPS \\
\hline Alimentos & 56.30 & 46.70 & 14.87 & 23.96 & 37.99 & 10.10 & 21.68 & 38.72 & 53.14 & 57.45 & 25.49 \\
\hline Textiles & 21.21 & 10.55 & 10.70 & 28.91 & 18.87 & 13.17 & 5.58 & 7.79 & 8.13 & 14.04 & 7.73 \\
\hline Madera & 1.25 & 4.62 & 1.19 & 0.99 & 4.33 & 2.20 & 1.38 & 0.48 & 4.96 & 3.08 & 2.07 \\
\hline Papel & 0.84 & 2.51 & 0.91 & 1.08 & 4.55 & 6.52 & 5.04 & 2.70 & 4.48 & 1.77 & 2.27 \\
\hline Químicos & 1.47 & 5.15 & 4.39 & 33.00 & 11.32 & 20.46 & 14.43 & 0.76 & 4.95 & 6.35 & 50.57 \\
\hline $\begin{array}{c}\text { Minería no } \\
\text { metálica }\end{array}$ & 3.56 & 3.93 & 9.26 & 4.47 & 7.38 & 6.81 & 12.28 & 3.76 & 11.22 & 3.73 & 3.25 \\
\hline Metálicos & 5.79 & 1.51 & 33.62 & 0.19 & 1.50 & 5.88 & 15.92 & 2.88 & 6.64 & 0.65 & 6.42 \\
\hline Maquinaria & 15.14 & 24.74 & 24.06 & 6.66 & 10.55 & 31.87 & 22.71 & 44.92 & 5.28 & 11.64 & 7.12 \\
\hline Otros & 0.17 & 1.77 & 1.00 & 0.73 & 3.52 & 3.00 & 0.97 & 0.88 & 1.21 & 1.27 & 1.43 \\
\hline
\end{tabular}

Fuente. Elaboración propia con datos del Banco de Información Económica del INEGI.

Tabla 8

\section{Cociente de localización de subsectores industriales} 1980

\begin{tabular}{|c|c|c|c|c|c|c|c|c|c|c|c|}
\hline & AGS & BC & COAH & GTO & JAL & MEX & NL & QRO. & SLP & SON & TAMPS \\
\hline Alimentos & 1.56 & 1.06 & 0.74 & 1.48 & 1.46 & 0.57 & 0.27 & 1.36 & 1.36 & 2.05 & 1.50 \\
\hline Textiles & 0.16 & 0.03 & 0.90 & 0.40 & 0.19 & 0.07 & 0.10 & 0.29 & 0.45 & 1.11 & 0.27 \\
\hline Papel & 1.30 & 1.29 & 1.00 & 0.97 & 1.07 & 1.29 & 0.90 & 1.18 & 1.41 & 1.12 & 1.46 \\
\hline Metálicos & 1.24 & 0.76 & 1.01 & 0.77 & 1.21 & 0.87 & 1.17 & 0.91 & 0.68 & 0.94 & 1.38 \\
\hline Maquinaria & 1.14 & 1.03 & 0.75 & 1.33 & 0.92 & 0.83 & 1.02 & 1.01 & 1.32 & 0.97 & 0.99 \\
\hline Otros & 0.92 & 1.24 & 0.90 & 0.83 & 0.87 & 0.68 & 0.97 & 0.79 & 0.93 & 1.00 & 1.02 \\
\hline
\end{tabular}

Fuente. Elaboración propia con datos del Banco de Información Económica del INEGI.

En 1980, Aguascalientes, Guanajuato, Coahuila, Querétaro, San Luis Potosí, Sonora y Tamaulipas, presentaban una mayor especialización en la industria alimentaria. Destacan Coahuila, estado de México y Nuevo León por tener una mayor diversificación en sus especializaciones. 
Se destacan fuertes especializaciones en la industria química en Baja California (1.90), Sonora (1.73) y Tamaulipas (1.98), la industria de la madera en el estado de México (1.66) y en Nuevo León (1.52), la industria alimentaria en Sonora (2.05). Pero como consecuencia de la integración regional del Bajío y de la apertura comercial, se presentan modificaciones en las especializaciones manufactureras.

Tabla 9

\section{Cociente de localización de subsectores industriales}

2014

\begin{tabular}{|c|c|c|c|c|c|c|c|c|c|c|c|}
\hline & AGS & BC & COAH & GTO & JAL & MEX & NL & QRO & SLP & SON & TAMPS \\
\hline Alimentos & 0.52 & 0.68 & 0.30 & 0.90 & 1.65 & 1.20 & 0.84 & 0.75 & 0.78 & 0.80 & 0.47 \\
\hline Textiles & 0.98 & 0.35 & 0.33 & 2.11 & 0.73 & 1.49 & 0.43 & 0.64 & 0.34 & 0.51 & 0.31 \\
\hline Madera & 0.19 & 0.33 & 0.11 & 0.16 & 0.39 & 0.25 & 0.26 & 0.34 & 0.19 & 0.21 & 0.13 \\
\hline Papel & 0.26 & 2.12 & 0.64 & 0.52 & 1.10 & 1.40 & 1.02 & 1.93 & 0.94 & 0.33 & 0.64 \\
\hline Químicos & 0.19 & 0.33 & 0.19 & 1.24 & 0.75 & 1.12 & 0.59 & 0.76 & 0.29 & 0.22 & 2.07 \\
\hline $\begin{array}{c}\text { Minería no } \\
\text { metálica }\end{array}$ & 0.40 & 0.76 & 1.20 & 0.92 & 0.72 & 0.61 & 1.18 & 0.77 & 1.99 & 0.75 & 0.45 \\
\hline Metálicos & 0.08 & 0.64 & 2.37 & 0.25 & 0.52 & 0.59 & 2.03 & 0.46 & 1.85 & 1.81 & 0.28 \\
\hline Maquinaria & 2.47 & 1.41 & 1.82 & 1.21 & 0.76 & 0.84 & 1.15 & 1.67 & 1.36 & 1.56 \\
\hline Otros & 0.44 & 5.08 & 0.30 & 0.24 & 1.70 & 1.38 & 0.65 & 0.17 & 0.32 & 0.97 & 1.40 \\
\hline
\end{tabular}

Fuente. Elaboración propia con datos del Banco de Información Económica del INEGI.

Para 2014, Aguascalientes presenta una amplia especialización en la industria de maquinaria con un cociente de localización de 2.47, Baja California tiene especialización otras industrias (5.08), en la industria del Papel (2.12) y de maquinaria (1.41), Coahuila se ha enfocado en la industria metálica (2.37) y de maquinaria (1.82), Guanajuato muestra mayor especialización en la industria de los textiles (2.11) y de maquinaria (1.21), Jalisco mantiene su mayor énfasis en la industria alimentaria (1.65) y en otras industrias (1.7), el estado de México presenta mayor especialización en la industria de los textiles (1.49) y el papel (1.4), Nuevo León se encuentra ampliamente enfocada a la industria metálica (2.03), Querétaro muestra especialización en la maquinaria (1.67) y en la industria del papel (1.93), San Luis Potosí tiene mayor enfoque en la industria de los minerales (1.99) y en la metálica (1.85), Sonora en la industria metálica (1.81) y en la de maquinaria (1.56) y por último, Tamaulipas tiene una amplia especialización en la industria química (2.07). 
Tabla 10

\section{Cociente de variación de subsectores manufactureros}

1980-2014

\begin{tabular}{|c|c|c|c|c|c|c|c|c|c|c|c|}
\hline & AGS & BC & COAH & GTO & JAL & MEX & NL & QRO & SLP & SON & TAMPS \\
\hline Alimentos & 3.41 & 1.70 & 3.29 & 7.04 & 2.91 & 4.79 & 2.72 & 3.02 & 1.99 & 2.21 & 1.92 \\
\hline Textiles & 2.82 & 0.65 & 0.83 & 2.28 & 0.43 & 0.76 & 0.91 & 2.11 & 0.93 & 0.97 & 0.71 \\
\hline Madera & 2.07 & 0.32 & 0.57 & 1.12 & 0.22 & 0.17 & 0.50 & 4.09 & 0.20 & 0.40 & 0.24 \\
\hline Papel & 11.75 & 10.07 & 11.51 & 9.18 & 1.65 & 0.88 & 1.44 & 11.28 & 2.87 & 3.01 & 3.01 \\
\hline Químicos & 31.00 & 4.98 & 4.59 & 4.70 & 2.96 & 1.48 & 1.92 & 104.76 & 5.25 & 3.64 & 2.86 \\
\hline $\begin{array}{c}\text { Minería no } \\
\text { metálica }\end{array}$ & 7.68 & 4.25 & 3.95 & 7.17 & 1.22 & 0.68 & 1.27 & 5.97 & 4.47 & 6.01 & 2.71 \\
\hline $\begin{array}{c}\text { Metálicos } \\
\text { Maquinaria }\end{array}$ & 207.29 & $1,957.64$ & 4.51 & 96.53 & 9.18 & 1.60 & 3.54 & 9.737 .39 & 14.77 & 175.47 & 177.60 \\
\hline Otros & 124.15 & 42.54 & 6.18 & 7.79 & 4.07 & 2.36 & 6.01 & 3.72 & 4.53 & 15.40 & 12.91 \\
\hline $\begin{array}{c}\text { Total } \\
\text { Región }\end{array}$ & 14.01 & 4.48 & 6.20 & 7.15 & 2.55 & 1.54 & 2.70 & 5.94 & 5.15 & 6.07 & 3.99 \\
\hline
\end{tabular}

Fuente. Elaboración propia con datos del Banco de Información Económica del INEGI.

Las nuevas especializaciones manufactureras han sido propiciadas por cambios en las tasas de crecimiento de los subsectores, ya que algunos han presentado mayor dinamismo. En la tabla 10, es posible analizar la variación que han presentado los subsectores de la industria manufacturera para las entidades federativas seleccionadas. Se observa un crecimiento extremadamente elevado en el subsector de la industria metálica y, de igual manera, la industria de maquinaria y equipo ha experimentado un fuerte crecimiento pero este se presenta en un número más reducido de estados.

Para concluir con el análisis de distribución territorial, se utiliza nuevamente el coeficiente de reestructuración, en el cual los valores positivos indican un cambio positivo en un subsector y signos negativos indican lo contrario. Se confirma lo que el resto de los indicadores muestran, que no sólo existe una nueva distribución del sector manufacturero en la zona bajío-norte del país, sino que también se ha dado un cambio en la estructura misma del sector industrial, las nuevas entidades federativas que lideran en este apartado han modificado su aparato productivo y se le ha destinado una mayor importancia principalmente a los subsectores de la industria de maquinaria y la industria metálica. 
Tabla 11

\section{Coeficiente de reestructuración de subsectores manufactureros}

1980-2014

\begin{tabular}{|c|c|c|c|c|c|c|c|c|c|c|c|}
\hline & AGS & BC & COAH & GTO & JAL & MEX & NL & QRO & SLP & SON & TAMPS \\
\hline Alimentos & -0.43 & -0.29 & -0.07 & 0.00 & 0.05 & 0.21 & 0.00 & -0.19 & -0.33 & -0.37 & -0.13 \\
\hline Textiles & -0.17 & -0.09 & -0.09 & -0.20 & -0.16 & -0.07 & -0.04 & -0.05 & -0.07 & -0.12 & -0.06 \\
\hline Madera & -0.01 & -0.04 & -0.01 & -0.01 & -0.04 & -0.02 & -0.01 & 0.00 & -0.05 & -0.03 & -0.02 \\
\hline Papel & 0.00 & 0.03 & 0.01 & 0.00 & -0.02 & -0.03 & -0.02 & 0.02 & -0.02 & -0.01 & -0.01 \\
\hline Químicos & 0.02 & 0.01 & -0.01 & -0.11 & 0.02 & -0.01 & -0.04 & 0.13 & 0.00 & -0.03 & -0.14 \\
\hline $\begin{array}{c}\text { Minería no } \\
\text { metálica }\end{array}$ & -0.02 & 0.00 & -0.03 & 0.00 & -0.04 & -0.04 & -0.07 & 0.00 & -0.01 & 0.00 & -0.01 \\
\hline Metálicos & 0.01 & 0.07 & -0.09 & 0.02 & 0.04 & 0.00 & 0.05 & 0.05 & 0.12 & 0.18 & 0.03 \\
\hline Maquinaria & 0.58 & 0.17 & 0.30 & 0.30 & 0.12 & -0.07 & 0.11 & 0.05 & 0.35 & 0.35 & 0.31 \\
\hline Otros & 0.01 & 0.15 & 0.00 & 0.00 & 0.02 & 0.02 & 0.01 & 0.00 & 0.00 & 0.02 & 0.03 \\
\hline Total Región & 0.62 & 0.43 & 0.31 & 0.32 & 0.25 & 0.23 & 0.18 & 0.25 & 0.48 & 0.55 & 0.37 \\
\hline
\end{tabular}

Fuente. Elaboración propia con datos del Banco de Información Económica del INEGI.

\section{Conclusiones.}

En México se ha presentado un cambio en la tendencia del crecimiento del sector manufacturero y estas modificaciones, impulsadas principalmente por distintas planes económicos a nivel federal y regional, han generado un nuevo patrón de localización y de especialización de los sectores productivos en las entidades federativas. Los cambios se generan en dos niveles. En el plano nacional, implican incrementos en general del sector manufacturero y de sus principales indicadores (índice de producto físico, productividad, empleo) y, en segundo lugar, estos nuevos cambios generan modificaciones significativas en la distribución de los sectores productivos que conforman el PIB de las entidades federativas.

Al carecer de una política industrial nacional, el desarrollo manufacturero en México se ha basado en la comercialización de productos a través de los tratados de libre comercio. Principalmente, las actividades industriales han aprovechado las ventajas que ofrece la situación geográfica de México al tener como vecino del norte al mercado más grande del mundo. La ausencia de barreras comerciales y una inmejorable localización ha permitido atraer inversiones extranjeras, las cuales se han traducido en posicionamientos industriales dentro de territorio mexicano.

Es necesario señalar que existen grandes ventajas y desventajas de la ejecución de esta política económica. En primer lugar, es realmente favorable aprovechar las facilidades con las que se cuenta, ya que el TLCAN ha permitido la instalación de numerosas industrias manufactureras en México, lo que 
ha provocado una importante derrama económica en los estados beneficiados. Todos estos resultados son fácilmente observables en el conjunto de técnicas mostradas en esta investigación. Sin embargo, ante la carencia de una política industrial articulada, México se encuentra desprotegido ante una crisis económica en Estados Unidos o ante una modificación en las reglas del libre comercio con América del Norte.

La conformación de regiones económicas es un aspecto con igual o mayor importancia unido a la necesidad de interrelacionar lo internacional, nacional y local dentro de un territorio (Florida, 2000). Así mismo, es necesario mantener un plan de desarrollo que trascienda gobiernos y que vincule al sector público, privado y académico (Vázquez, 2002).

Ante estos nuevos paradigmas las regiones de México han reaccionado de forma diferente. Las entidades del norte del país han basado su estrategia industrial en su favorable localización geográfica, por lo que la reducción en costos de transporte y la sencilla movilización hacia los Estados Unidos parten como sus principales ventajas comparativas frente al resto de los estados mexicanos.

La región del Bajío se ha enfocado en mayor medida en seguir los nuevos paradigmas de la localización industrial y cambio estructural. En este territorio se ha creado una integración regional a través de sistemas de planeación estatal, los cuales se encuentran vinculados con el resto de las entidades federativas que conforman la región. Así mismo, se ha dado una interacción constante entre los tres grandes actores del sistema económico, sector público, privado y académico (Pérez, 2015).

Ante las nuevas configuraciones industriales resultantes de la apertura comercial y a la entrada de IED en territorio mexicano, es posible generar una clasificación en el nivel de crecimiento del producto interno bruto (PIB). En primer lugar, Aguascalientes y Querétaro son los estados sobresalientes, presentando tasas de crecimiento del PIB de 6 por ciento. Otras entidades ganadoras son Coahuila, Nuevo León y Guanajuato. En segundo lugar, se encuentran los estados con crecimiento intermedio, entre los que se encuentran Tamaulipas, Tlaxcala, Yucatán y Sonora. Entre las entidades que su crecimiento es moderado están Puebla, Jalisco, Michoacán y Baja California Sur. Por último, Sinaloa, Hidalgo, Chiapas y Morelos son los estados perdedores (Gasca, 2005).

De igual manera, De León-Arias y Parra-De la Torre (2011) exploran las características de la dinámica de la productividad manufacturera por entidad federativa en México, logrando identificar tres grupos de entidades en función de su desempeño productivo: uno de las ganadoras, otro de las perdedoras y un tercero conformado por el resto del país. Tales entidades permitieron a los investigadores ubicar el patrón de crecimiento en los estados de la frontera norte, el centro occidente y el oriente mientras que el gran perdedor resultó ser la Ciudad de México.

Cabe señalar que en el presente artículo es posible generar una clasificación propia a partir de los resultados obtenidos en las TAR, donde se muestra la nueva localización industrial y las nuevas especializaciones manufactureras. 
Se tiene que las entidades federativas que conforman las regiones del norte y del Bajío son las ganadoras en materia industrial, destacándose principalmente Coahuila, Aguascalientes, Querétaro, Sonora y Guanajuato.

En segundo lugar, es posible identificar aquellas entidades federativas que han presentado un desempeño aceptable en su transformación productiva hacia la industria, se tienen en esta categoría a los estados de Jalisco, San Luis Potosí y Tamaulipas principalmente.

Los estados perdedores son aquellos en los que las manufacturas han perdido peso en su estructura productiva y donde se mantienen, en cierta medida, las mismas especializaciones industriales desde hace 30 años, se destacan en esta categoría las entidades de Puebla, Morelos, Estado de México y Ciudad de México.

Por último, se tiene aquellas entidades en las que la industria no ha ganado ni perdido peso, simplemente estos estados se encuentran especializados en otras ramas productivas y no dan gran importancia a la industria manufacturero, se destacan los estados que conforman la región sur del país, acompañados por Colima, Nayarit, Baja California Sur y Sinaloa, entre otros.

Sin duda que los resultados obtenidos evidencian el proceso de trasformaciones vividas en México desde principios de la década de los ochentas con especial énfasis en la liberalización del comercio a mediados de la década de los noventas del siglo pasado.

Como se ha señalado, desde mediados del siglo anterior, nuestro país había adoptado un modelo al que le distinguía una excesiva centralización del aparato productivo, que era la expresión de una estrategia orientada a la sustitución de exportaciones y a la que le caracterizaban políticas proteccionistas y procesos de inversión impulsados y orientados por el Estado.

Pero en un nuevo contexto mundial, con una nueva política comercial con orientación hacia afuera, la industria encontró mayores posibilidades para su desarrollo aunque con características muy particulares. En el proceso, la presencia de una mayor dotación de infraestructura en ciertas regiones, un esquema de exención de impuestos y un rol preponderante de la IED, redefinió la localización de las regiones industrializadas en el plano nacional y el perfil de las exportaciones nacionales.

Lo que en este contexto también parece quedar claro, es que el proceso de apertura comercial no contemplaba en forma explícita resolver las disparidades regionales del país, sino que en todo caso la nueva dinámica que habría de caracterizarle sería capaz de impactar en positivo en aquellas regiones que por las características propias del modelo previo se han habían mantenido marginadas. Lo que se advierte es que en todo caso esta expectativa si acaso se habrá cumplido parcialmente, en el sentido de que si bien efectivamente regiones como la del Bajío parecen haber resultado beneficiadas, otras como las del sur no acusan ni más leve mejoría en el periodo.

Por otra parte, no obstante que el desarrollo de la actividad manufacturera en el país le ha permitido convertirse en el primer exportador de manufactura en América Latina para ubicarse así 
como un actor relevante en las cadenas productivas globales de valor, lo cierto es que al revisar las características y el destino de las exportaciones manufactureras, así como el origen de los capitales que financian la mayor parte de ellas, se advierte un alto grado de fragilidad para la economía mexicana, en tanto se aprecia sujeta a las oscilaciones de la economía internacional y con un alto grado de dependencia de las crisis financieras a nivel mundial y lo que es más grave aún, en ausencia de una política de industrialización nacional. 


\section{Bibliografía y referencias documentales}

Aydalot, Phillipe. (1985). Economía regional y urbana. Editions Economica.

Boisier, Sergio. (1980). Técnicas de análisis regional con información limitada. Cuadernos del ILPES.

Castañeda, Alejandro. (2010). El tratado de libre comercio de américa del norte. Los grandes problemas de México. El Colegio de México, A.C.

Christaller, Walter. (1933). Central Plances in Southern Germany. Prentice Hall.

Cordera, Rolando. (2015). "La "Gran Transformación” del Milagro Mexicano. A 20 años del TLCAN: de la adopción a la adaptación". Problemas del desarrollo, 46(180), 11-25.

De León-Arias, Adrian. y Parra de la Torre, Edna (2011). "Crecimiento Económico en las manufacturas mexicanas: un análisis de contabilidad del crecimiento en las entidades federativas, 1988-2003". Economía, Sociedad y Territorio, 575-607.

Esqueda, Ramiro. (2013). "Economías de aglomeración en el contexto de la nueva geografía económica". Contribuciones a la Economía. Recuperado de www.eumed.net/ce/2013/economias-aglomeración. html.

German-Soto, Vicente., Escobedo, J y Flores, Luis (2010). "Convergencia y divergencia en el sector industrial de los estados mexicanos: un análisis espacial no paramétrico". Equilibrio Económico, 6(2), 161-186.

Greenhut, M. L. (1957). Games, Capitalism and General Location Theory. Manchester School of Economic and Social Studies.

Hayter, Rogert. (1997). The dynamics of industrial location: the factory, the firm and the production system. Wiley.

Hernández, I. (2007). Liberalización comercial y localización industrial en México. Barcelona.

Holmes, Thomas. (2003). Spatial Distribution of Economic Activities in North America. University of Minnesota and Federal Reserve Bank of Minneapolis and National Bureau of Economic Research.

Hoover, Edgar. (1948). The Location of Economic Activity. McGraw-Hill.

Hoover, Giarranti. (1985): An Introduction to Regional Economics, 3. ${ }^{a}$ ed., Alfred A. Knopf, New York.

Huerta, Heliana.,y Chávez, Maria (2000). "Tres modelos de política económica en México durante los últimos sesenta años”. Red Análisis Económico. vol. 18, no 37.

Isard, Walter. (1998). Methods of Interregional and Regional Analysis, MIT.

(1960). Methods of Regional Analysis. MIT, 1960, pp.249ff.

Krugman, Paul. y Livas, Raul. (1992). "Trade Policy and the Third World Metropolis”, National Bureau of Economic Research Working Paper No. 4238.

Leontief, Wassily. (1951). The estructure of American Economy, 1919-1939, Oxford University Press.

Lira, Cossio., y Quiroga, Bolivar. (2003). Técnicas de análisis regional. United Nations Publications. Vol. 30.

Losch, August. (1940). The Economics of Location. Yale University Press

Marshall, Alfred. (1920). Principios de economía. Un tratado de introducción. Madrid: Aguilar.

Méndez, Elier., y Lloret, Maria. (2004). Las técnicas de análisis regional como parte del diagnóstico en proceso de panificación regional en cuba. Cuba: Observatorio de la economía latinoamericana

Mori, Tomoya. Nishikimi, Koji. y Smith, Tony. (2005). “A Divergence Statistic for Industrial Localization. The Review of Economics and Statistics, 87(4), 635-651.

Mendoza José. y Pérez, Jorge. (2007). "Efectos de la aglomeración y los encadenamientos industriales en el patrón de crecimiento manufacturero en México". Investigaciones Regionales, 109-133.

Ohlin, Bertil. (1933). International and interregional trade. Harvard Economic Studies, Cambridge, MA. 
Relocalización de la industria manufacturera en México... • Jorge Rafael Figueroa Elenes, Tomás Arroyo Parra y Aneliss Aragón Jiménez

Pasinetti, Luigi. (1973). "The notion of vertical integration in Economic analysis". Metroeconomica, 25. Pp. $1-29$.

Shaver, Myles. (2015). "Agglomeration economies, firm heterogeneity, and foreign direct investment in the united states". Strategic Managment Journal. Vol.21(12), 1175-1193

Sraffa, Piero. (1960). Production of ecommodities by means of commodities. Prelude to a critique of Economic theory. Cambridge, Cambridge University Press.

Ruiz, Clemente. (2015). Reestructuración productiva e integración. tlcan 20 años después. Problemas del desarrollo, 46(180), 27-50.

Von Thunen, Johan. (1820). El estado aislado.

Weber, Alfred. (1909). Theory of the Location of Industries. Chicago. 
Números anteriores:

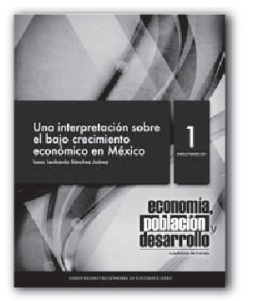

Economía, población y desarrollo.
Cuadernos de trabajo №1

Enero-Febrero 2011
Una interpretación sobre el bajo

crecimiento economico en México
Isaac Leobardo Sánchez Juárez

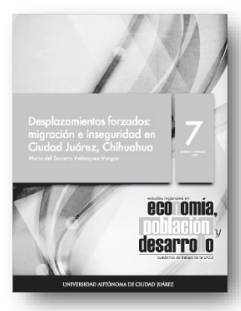

Economia, población y desarroll

Cuadernos de trabajo $\mathrm{N}$ -
Encro-Fcbrcro 2012

Desplazamientos forzados
migración e inseguridad

Ciudad Juárez, Chihuahua
Maria del Socorro Velázquez Varga

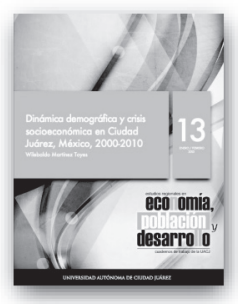

Economiá, población y desarrollo

de trabajo $N$

Dinámica demográ́fica y crisis
socieconómica en Ciudad Juáre Mexico, 2000-2010

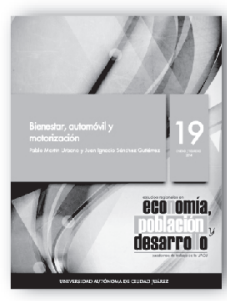

Economía, población y desarroll Enero - Febrero 2014 Bienestar, automóvil y motorización
Pablo Martín Urbano y Miosánchez Gutiérrez

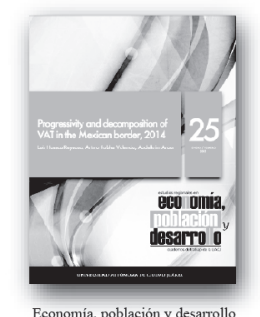

Cuadernos de trabajo No $_{0}$
Conomia,

Encro - Febrero 2015

Progressivity and decomposition of
VAT in the Mexican border, 2014 Abdelkim Araar

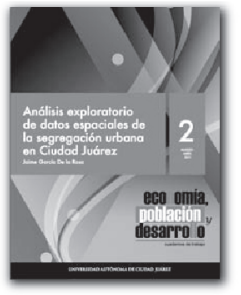

Economía, población y desarrollo.
Cuadernos de trabajo № 2

Marzo-Abril 2011

espaciales de la segregacón

Jaime Garcia De la Rosa

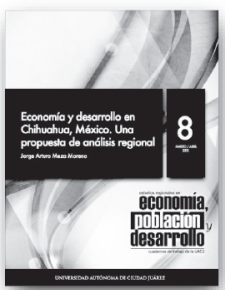

Economia, población y desarrollo

Cuadernos de trabajo №7

Economía y desarrollo en

propuesta de análisis regiona

Jorge Arturo Meza Moreno

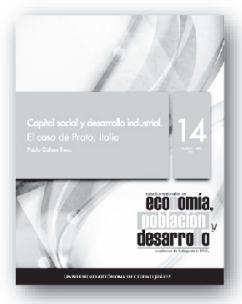

Economía, población y desarrollo

Marzo - Abri1 2013

Capital social y desarrollo

Pablo Galaso Reca

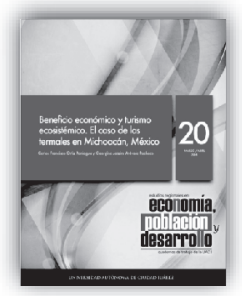

Economia, población y desarrollo

Marzo - Abril 2014

Beneficio económico y turismo

enico. El caso de las ter
en Michoacán, Mésico

arlos Franciseo Ortiz Paniagu

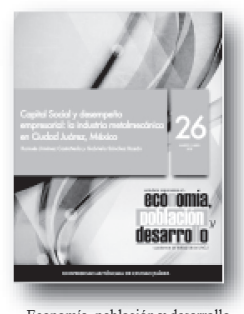

Economía, población y desarrollo

Cuadernos de trabajo № 26
Marzo - Abril 2015

Capital Social y desempeño empresa
la industria metalmecánica en

a industria metalmecánica en
Ciudad Juárez, México

Ramsés Jiménez Castañeda y
Gabriela Sáncez Bazán
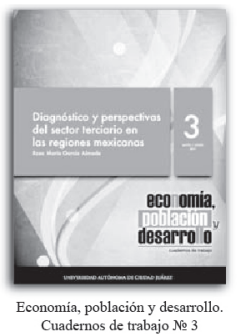

Diagnóstico y perspectivivas

regiones mexicanas
rosa Mária Garcia Almad

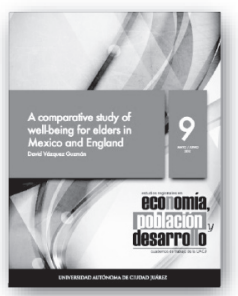

Economia, población y desarrollo

Cuadernos de trabajo № 9
Mayo - Junio 2012

A comparative study of

Mexico and England
David Vázquez Guzmán

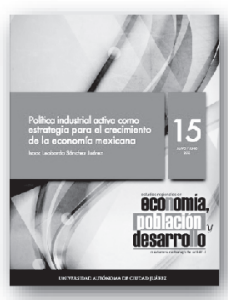

Economia, población y desarroll

Madernos de trabajo
Mayo - Junio 2013

Politica ind ustrial activa como

strategia para el crecimiento

Isaac Lcobardo Ś́nexicana
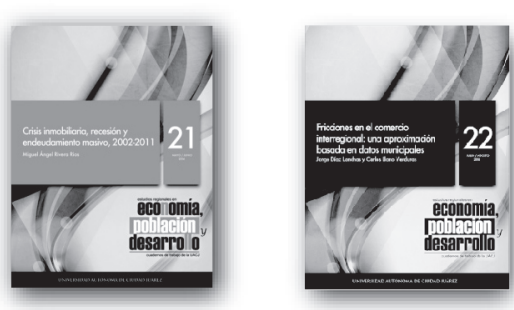

Economia, población y desarrollo

Cuademos de trabajo
Mayo - Junio 2014

Crisis inmobiliaria, recesión y
adeudamiento masivo, $2002-2011$

Miguel Ángel Rivera Rios

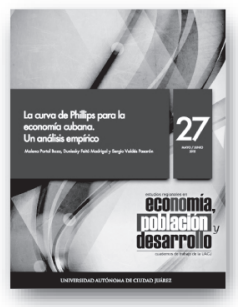

Economia, población y desarrollo

Cuadernos de trabajo № 27

a curva de Phillips para

economía cubana.

Malena Portal Boza, Duniesky Feitó

Economía, población y desarrollo.
Cuadernos de trabsio

julio-Agosto 201

Los indices IDH y FGT en la

mera década del siglo XX

Economía, población y decarrollo

Cuadernos de trabajo №
Julio - Agosto 2012

Political competition and the

edistribution in a federation

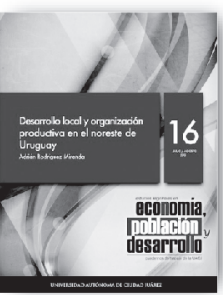

Economía, población y desarrollo

Cuadernos de trabajo №
Julio - Agosto 2013

Desarrollo local y organización
productiva en el noroeste de Urugu

Economía, población y desarrollo

Julio - Agosto 2014

Ficciones en el comercio

basada en datos municipales
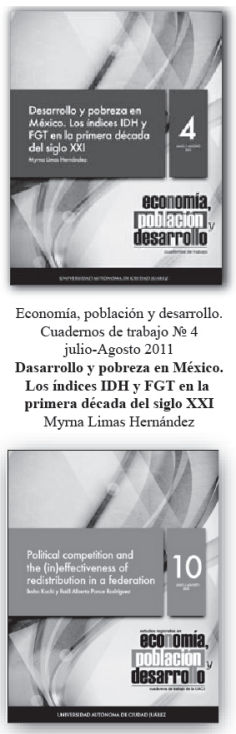

Jorge Diaz Lanchas y Carlos Llano Verduras
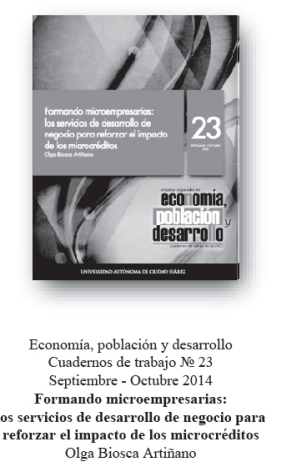

Cuadernos de trabajo № 24

El crecimiento de las regiones

el paradigma del desarrollo

divergente. Un marco térico
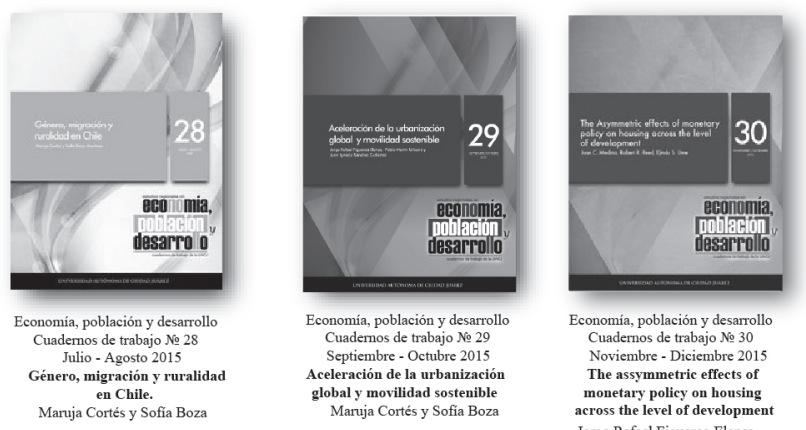

conomía, población y desarrol

Cuadernos de trabajo № 30

The assymmetric effects of

monetary policy on housing

Jorge Rafael Figueroa Elenes,

Juan Ignacio Sánchez Gutiérrez 
Números anteriores:
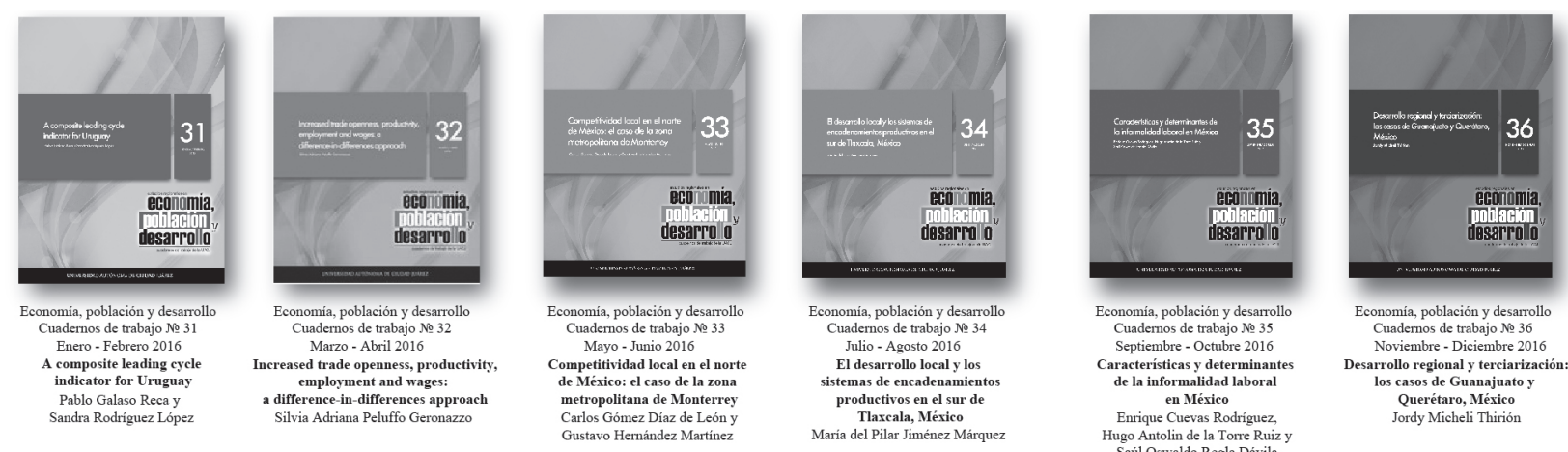

conomía, población y desarrollo Septiembre - Octubre 2016

Caracteristicas $y$ determinantes
de la informalidad laboral en México

Enrique Cucvas Rodrígucz,
Hugo Antolin de lo Torre Ruz

Gustayo Herrández Martinez

Maria del Pilar Jiménez Márquez
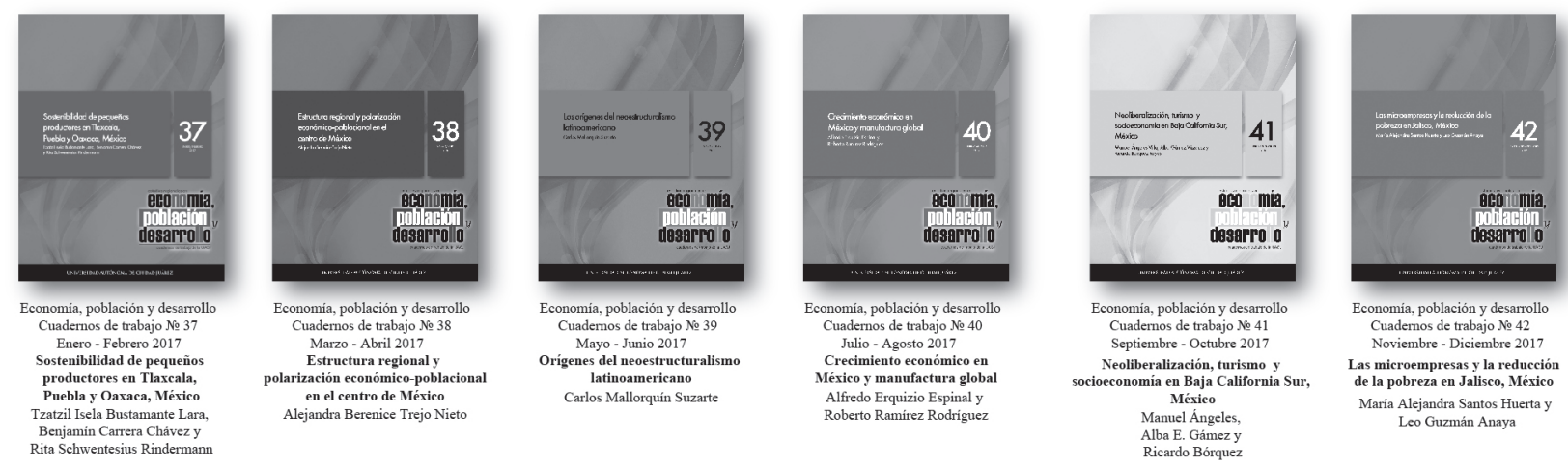

Cuadernos de trabajo № 42 Noviembre - Diciembre 2017 Enero - Febrero 2017 productores en Thaxcala, Puebla y Oaxaca, México
zatzil Iscla Bustamante Lar Benjamin Carrera Chávez y
Rita Schwentesius Rindermant

en el centro de México latinoamericano
Carlos Mallorquin Suzarto

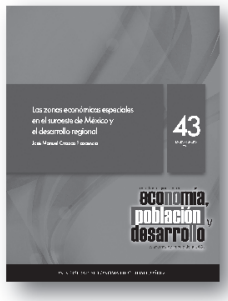

Economia, población y desarrol Cuadernos de trabajo 1040
Enero - Febrero 2018

Encro - Febrero 2018
Las zonas económicas especiales el desarrollo regional José Manuel Orozco Plascencis 


\section{$\rightarrow$ Normas Editoriales}

\section{Para el documento general:}

Tipo de letra: Times New Roman.

Tamaño: 11 puntos.

Interlineado: 1.5 espacios.

Títulos y subtítulos:

El texto principal en 11 puntos. Títulos 12 puntos (en resaltado). Subtítulos 11 puntos. Cada título y subtítulo deberá numerarse bajo el siguiente orden: $1,1.1,2,2.1,2.2 \ldots$

La extensión máxima de los cuadernos de trabajo será de 40 cuartillas.

La primera vez que se emplee una sigla en el texto se especificará primero su equivalencia completa y después la sigla.

\section{Hoja de presentación:}

Título:

14 puntos, centrado, resaltado.

Nombre de autor(es):

12 puntos

Resumen y abstract:

Debe incluir resumen en español y abstract (diez puntos), no mayor a 250 palabras

Palabras clave:

Incluir entre tres y cinco palabras clave, en español e inglés

Referencia del autor o autores:

Institución de adscripción, grado académico y líneas-grupos de investigación que desarrolla y a los que pertenece.

\section{Sistema de referencia de citas:}

Harvard-APA

Las citas bibliográficas en el texto deberán incluir entre paréntesis sólo el apellido del autor, la fecha de publicación y el número de página; por ejemplo: (Quilodrán, 2001: 33).

\section{Notación en sección de bibliografía y fuentes de información:}

Se deberá incluir al final del texto. Toda referencia deberá estar mencionada en el texto o notas de pie de página.

Cada referencia iniciará con el primer apellido o los apellidos, luego el nombre del autor, y después, entre paréntesis, el año de publicación seguido de un punto. Ejemplos:

Se deberá incluir al final del texto. Toda referencia deberá estar mencionada en el texto o notas de pie de página.

Cada referencia iniciará con el primer apellido o los apellidos, luego el nombre del autor, y después, entre paréntesis, el año de publicación seguido de un punto. Ejemplos:

Artículo:

Ros, Jaime (2008). "La desaceleración del crecimiento económico en México desde 1982”, en Trimestre Económico, vol. 75, núm. 299, pp. 537-560.

Libro:

Villarreal, René (2005). Industrialización, competitividad y desequilibrio externo en México. 
Un enfoque macroindustrial y financiero (1929-2010), México, Fondo de Cultura Económica. Capítulo de libro:

Castillo, Manuel Ángel (2003). "La política de inmigración en México: un breve recuento", en Manuel Ángel Castillo, Alfredo Lattes y Jorge Santibáñez (coords.), Migración y fronteras, Tijuana, El Colegio de la Frontera Norte / Asociación Latinoamericana de Sociología / El Colegio de México, pp. 425-451.

\section{Notas de pie de página:}

Se utilizarán para hacer indicaciones complementarias, aclaraciones o ampliación de una explicación. La notas de pie de página en Times New Roman, 10 puntos.

\section{Tipología de imágenes dentro del texto:}

Cuadro

Gráfica

Diagrama

Mapa

Figura

Todas las imágenes deben ser numeradas y mencionadas dentro del texto. A toda imagen debe incluirse la fuente.

Las indicaciones de la imagen: tipo y número de imagen, título de imagen y fuente se escriben en 10 puntos. En el texto poner como imagen los mapas, figuras, gráficas y diagramas -con el ánimo de no perder el formato realizado por el autor.

\section{Ecuaciones y fórmulas:}

Si se utilizan ecuaciones o fórmulas deberá utilizarse el editor de ecuaciones de Word y numerarse.

\section{Envío de trabajos}

Los trabajos deben ser enviados a la dirección de correo: lgtz@uacj.mx. Con el Dr. Luis Enrique Gutierrez Casas, editor de esta publicación.

La aceptación de cada colaboración dependerá de la evaluación de dos dictaminadores especialistas en la materia que se conservarán en el anonimato, al igual que el autor (autores) para efectos de la misma. 


\section{$\rightarrow$ Editorial Guidelines}

\section{For General Document:}

Font type: Times New Roman.

Size: font size 11 .

Paragraph: 1.5 line spacing.

Titles and subtitles: Main text font size 11. Titles font size 12 (Bold). Subtitles font size 11.

Each title and subtitle should be numbered in the following order: 1, 1.1, 2, 2.1, 2.2...

The maximum length of the workbooks will be 40 pages.

The first time an abbreviation is used in the text will be specified first complete equivalence and then stands.

\section{Front cover:}

Title:

Font size 14, centered, Bold.

Author name(s):

Font size 12.

Abstract:

It should include abstract in Spanish and abstract (font size 10), no more than 250 words.

Keywords:

Include three to five keywords, in Spanish and English.

Reference of author:

Institution of affiliation, academic degree and line-developed by research groups and belonging.

\section{Bibliographical appointment system:}

Harvard-APA

Citations in the text should include between parentheses only the author's name, publication date and page number, for example:

(Quilodrán, 2001: 33).

\section{Notation about Bibliography section and Information fonts:}

Should be included at the end of the text. All references must be mentioned in the text or footnotes page.

Each reference starts with the first name or last name, then the name of the author, and then, in parentheses, the year of publication followed by a period. Examples:

Article:

Ros, Jaime (2008). “La desaceleración del crecimiento económico en México desde 1982”, en Trimestre Económico, vol. 75, núm. 299, pp. 537-560.

Book:

Villarreal, René (2005). Industrialización, competitividad y desequilibrio externo en México. Un enfoque macroindustrial y financiero (1929-2010), México, Fondo de Cultura Económica.

Book chapter:

Castillo, Manuel Ángel (2003). "La política de inmigración en México: un breve recuento”, en Manuel Ángel Castillo, Alfredo Lattes y Jorge Santibáñez (coords.), Migración y fronteras, Tijuana, E1 Colegio de la Frontera Norte / Asociación Latinoamericana de Sociología / El Colegio de México, pp. 425-451. 


\section{Footnotes:}

Must be used to make additional indications, clarification or expansion of an explanation. The footnotes must be in Times New Roman, font size 10.

\section{Image typology inside text:}

Picture

Graph

Diagram

Map

Figure

All images must be numbered and mentioned in the text, should include the source image. The indications of the image: type and number of image, image title and source are written in 10 font size. In the text set as image maps, figures, graphs and charts-with the intention of not losing the formatting by the author.

\section{Equations and Formulae:}

When using equations or formulas should be used in Microsoft Word equation editor and numbered.

\section{Paper sending}

Entries must be sent to the email address: lgtz@uacj.mx. With Dr. Luis Enrique Gutiérrez Casas, editor of this publication.

Acceptance of each collaboration will depend on the evaluation of two examiners skilled in the art to be kept anonymous, like the author(s) for the same purposes. 


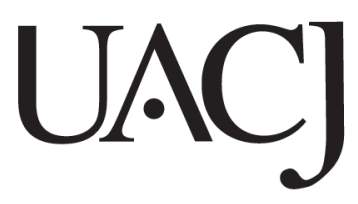

Esta obra se terminó de imprimir en febrero de 2018 Cd. Juárez, Chihuahua, México.

Tiraje: 120 ejemplares 
Cuadernos de Trabajo de la Universidad Autónoma de Ciudad Juárez, número 44, marzo - abril de 2018

\section{Director y editor}

Dr. Luis Enrique Gutiérrez Casas

\section{Comité editorial}

Sección internacional

Dra. Sofía Boza Martínez (Universidad de Chile, Chile)

Dra. Olga Biosca Artiñano (Glasgow Caledonian University, Reino Unido)

Dra. Ángeles Sánchez Díez (Universidad Autónoma de Madrid, España)

Dr. Thomas Fullerton Mankin (University of Texas at El Paso, Estados Unidos)

Dr. Adrián Rodríguez Miranda (Universidad de la República, Uruguay)

\section{Sección local}

(Universidad Autónoma de Ciudad Juárez)

Dra. Myrna Limas Hernández

Dra. Ikuho Kochi

Dr. Raúl Alberto Ponce Rodríguez

Dr. Isaac Leobardo Sánchez Juárez

Dr. Héctor Alonso Barajas Bustillos

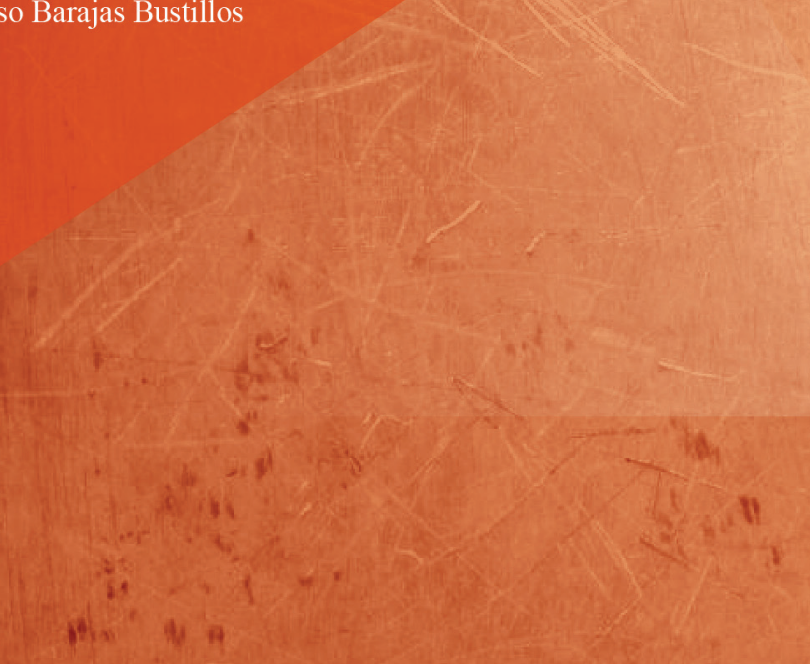

Universidad Autónoma de Ciudad Juárez

Instituto de Ciencias Sociales y Administración

Cuadernos de Estudios Regionales en Economía, Población y Desarrollo ISSN 2007-3739

Cuerpo Académico de Estudios Regionales en Economía, Población y Desarrollo

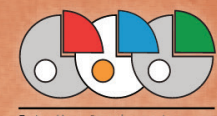

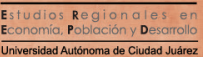

www.estudiosregionales.mx

Publicación afiliada a la Red Iberoamericana de Estudios del Desarrollo

C Universidad Autónoma de Ciudad Juárez Avenida Plutarco Elías Calles \#1210, Fovissste Chamizal Ciudad Juárez, Chih., México. www.uacj.mx 